\title{
MedienPädagogik
}

Zeitschrift für Theorie und Praxis der Medienbildung

\section{Aufwachsen in mediatisierten Lebenswelten}

\section{Zur Notwendigkeit des Zusammenwirkens von Medienpädagogik und Medienschutz}

\author{
Dieter Spanhel
}

\section{Zusammenfassung}

Im Folgenden wird die These vertreten, dass unter den Bedingungen mediatisierter Lebenswelten ein gedeihliches Aufwachsen mit Medien weder allein durch Massnahmen des Kinder- und Jugendmedienschutzes, noch allein durch eine umfassende Medienkompetenzförderung gesichert werden kann. Um die Notwendigkeit für ein abgestimmtes Zusammenwirken zwischen den Institutionen des Medienschutzes und der Medienpädagogik zu begründen, muss das Medienhandeln der Heranwachsenden in den mediatisierten Kontexten ihrer Alltagswelt in Familie, Kita, Schule und Freizeit aus einer systemtheoretischen Perspektive analysiert werden. Aus dieser Sicht ist ihr Medienhandeln sinnorientiert und als Ergebnis innerpersonaler Regulationsprozesse zu verstehen. Es findet in Form medialer Kommunikationsprozesse statt, in denen die psychischen Systeme der Heranwachsenden und die für sie relevanten sozialen Systeme wechselseitig aufeinander einwirken. Dabei bilden sich Kommunikationsmuster aus, in denen die Heranwachsenden ihre eigenen Bedürfnisse, Interessen und Ziele zu verfolgen suchen, aber auch den Erwartungen, Normen und Regeln der sozialen Systeme gerecht werden müssen. Durch eine Analyse der Muster und Regeln dieser kommunikativen Austauschprozesse werden die Faktoren aufgedeckt, die auf das 
selbstregulierte Medienhandeln der Heranwachsenden einwirken. Dabei wird sichtbar, welche notwendigen, aber gleichwohl begrenzten Einflussmöglichkeiten sowohl Medienschutz als auch medienpädagogische Massnahmen in diesem Faktorengeflecht haben. Auf dieser Basis lassen sich Notwendigkeit und Möglichkeiten ihres konzertierten Zusammenwirkens für bessere Bedingungen des Aufwachsens aufweisen. Als Grundlage für ein Modell medienpädagogischen Handelns, das Schutzfunktion und pädagogische Unterstützung verbindet, wird das Konzept einer kontextuellen Steuerung des Medienhandelns der Heranwachsenden entwickelt. Es beruht auf der Gestaltung der Sinn stiftenden Kontexte für ihr Medienhandeln in Familie, Kita, Schule und Freizeit. Nur in einem Austarieren von Grenzsetzungen, Autonomiegewährung und medienpädagogischer Begleitung eröffnen sich den Kindern und Jugendlichen in diesen Kontexten Spielräume, in denen sie lernen, persönliche und soziale Sinnorientierungen aufeinander abzustimmen und so ihr Medienhandeln eigenverantwortlich zu steuern. Für die praktische Umsetzung werden Prinzipien, Ansatzpunkte und konkrete Beispiele für die schwierige Aufgabe einer kontextuellen Steuerung des Medienhandelns aufgezeigt. Diese steht nicht im Widerspruch zur Selbstregulation psychischer und sozialer Systeme, bietet aber immerhin eine reelle Chance, den Entwicklungsprozess der Kinder und Jugendlichen unter den Bedingungen sich rasch wandelnder mediatisierter Lebenswelten an den Sinnkriterien intellektueller und moralischer Autonomie und der Befähigung zu verantwortlicher sozialer Teilhabe auszurichten.

\title{
Growing up in mediatized worlds. On the need for interaction between media education and media protection
}

\begin{abstract}
In this paper, the thesis is put forward that under the conditions of mediatized living environments, successfully growing up with media can neither be ensured solely through measures to protect children and young people from the media, nor solely through comprehensive media competence promotion. In order to justify the need for a coordinated interaction between the institutions of media protection and media education, the media behavior of adolescents in
\end{abstract}


the mediatized contexts of their everyday world in family, daycare, school and leisure time must be analyzed from a system-theoretical perspective. From this point of view, media actions are meaning-oriented and should be understood as the result of internal regulation processes. They take place in the form of media communication processes in which the psychological systems of the adolescents and the social systems relevant to them interact with one another. Communication patterns develop in which the adolescents seek to pursue their own needs, interests and goals, but also have to meet the expectations, norms and rules of the social systems. By analyzing the patterns and rules of these communicative exchange processes, the factors that affect the self-regulated media behavior of adolescents are revealed. In doing so, it becomes clear which necessary but limited possibilities of influence both media protection and media educational measures have in this network offactors. On this basis, the necessity and possibilities of their cooperation for better conditions for growing up can be demonstrated. As a basis for a model of media educational action that combines protective function and educational support, the concept of contextual control of media actions of adolescents is being developed. It is based on the creation of meaningful contexts for media activities in family, daycare, school and leisure time. Balancing boundaries, autonomy and media-pedagogical accompaniment may give children and young people the room, in which they learn to coordinate personal and social orientations of meaning and thus to control their media actions independently. For practical implementation, principles, starting points and examples for the difficult task of contextual control of media activity are shown. This does not contradict the self-regulation of psychological and social systems, but at least offers a chance to improve the development process of children and adolescents under the conditions of rapidly changing mediatized worlds in terms of the meaningful criteria of intellectual and moral autonomy and the ability to be socially responsible.

\section{Einleitung}

Die Bedingungen des Aufwachsens unserer Kinder und Jugendlichen haben sich in der Folge des tiefgreifenden Medienwandels in den vergangenen Jahrzehnten radikal verändert. Von Geburt an werden die Kinder in allen Lebensräumen mit digitalen Medien konfrontiert und Medien wirken 
beim Vollzug der verschiedensten Lebensformen mit. Der Medienalltag der Kinder und Jugendlichen ist heute dadurch gekennzeichnet, dass sie auf ihren mobilen Endgeräten über die ganze Fülle und Breite faszinierender Medienangebote ohne Schwierigkeiten verfügen können. Schon von klein auf nutzen sie die digitalen Angebote immer weniger nur als Rezipierende, sondern als aktiv handelnde Subjekte. Bei der Gestaltung ihrer Alltagswelt gebrauchen sie die Medien kompetent als Informations- und Kommunikationsmittel, zur Teilhabe an sozialen und kulturellen Netzwerken, zur Gestaltung ihrer Freizeit, zum Aufenthalt in virtuellen Welten, zur Selbstdarstellung und Präsentation eigener Werke, zur Reflexion ihres Denkens und Handelns und damit zur Entwicklung ihrer Identität. Diesen Lern-, Entwicklungs- und Bildungschancen stehen auf der anderen Seite vielfältige Lernzwänge, Besorgnis erregende Entwicklungsbeeinträchtigungen und Verletzungen der Grundrechte der Kinder gegenüber. In der Öffentlichkeit wird einerseits der mangelnde Schutz der Heranwachsenden vor den Entwicklungsgefährdungen durch die Medien beklagt, andererseits wird die unzureichende Vermittlung von Medienkompetenz in den Bildungseinrichtungen kritisiert.

Aus anthropologischer Sicht verändern diese Bedingungen des Aufwachsens in einer mediatisierten Lebenswelt ganz grundlegend die Beziehungen der Heranwachsenden zur Welt, zu den Mitmenschen und zu sich selbst. Wie ist unter diesen Bedingungen ein gelingendes Aufwachsen möglich? Wie können Kinder und Jugendliche in ihrer Entwicklung einen Bildungsprozess durchlaufen, der sie befähigt, ihr Menschsein selbstbestimmt zu verwirklichen und gleichzeitig zur Erhaltung und Weiterentwicklung der Gesellschaft beizutragen? (Spanhel 2010a; 2014; Kammerl und Kramer 2016)

Als Antwort auf die Folgen der tief greifenden Medienentwicklungen für die Heranwachsenden haben sich bereits im 20. Jahrhundert die Institutionen des Kinder- und Jugendmedienschutzes einerseits und der Medienpädagogik andererseits herausgebildet. Der Kinder- und Jugendmedienschutz wurde als Staatsaufgabe gesetzlich verankert (Baum 2008) mit dem Ziel, die Medienindustrie, die Medienanbieter und Provider in die Verantwortung zu nehmen, um die Heranwachsenden vor entwicklungsgefährdenden und die Würde der Person verletzenden Angeboten zu schützen. 
Die Medienpädagogik kämpft seit den 1970er Jahren um die gesellschaftliche Anerkennung der Medienbildung als fundamentale Erziehungs- und Bildungsaufgabe und ihre Verankerung in den Erziehungs- und Bildungseinrichtungen (Spanhel 2015). Sie wendet sich an die Verantwortung der Eltern, Erziehende und Lehrpersonen, für alle Heranwachsenden die Rahmenbedingungen für ein gedeihliches Aufwachsen zu gestalten und sie zu einem selbstbestimmten, sinnvollen, reflexiv-kritischen und sozial verantwortlichen Medienumgang zu befähigen.

Beide Institutionen - das wird in jüngster Zeit nun immer deutlicher können in der Folge der rasanten Weiterentwicklung der Medien durch die Entwicklung der Digitalisierung, Vernetzung und Informations- und Kommunikationstechniken ihren Aufgaben nicht mehr angemessen eigenständig nachkommen. Das zeigen Forderungen nach einer Ausweitung der $\mathrm{Me}$ dienpädagogik in Richtung auf digitale Bildung durch Einbeziehung informationstechnischer Bildungsinhalte und die dringliche Einforderung neuer Schutzmassnahmen im Zusammenhang mit Digitalisierung und Internet, z. B. Datenschutz, Persönlichkeitsschutz, Schutz der Privatsphäre oder Vertrauen und Sicherheit im Internet (SINUS-Institut Heidelberg 2015). Das wird auch deutlich in den kontroversen Diskussionen um die Novellierung der gesetzlichen Grundlagen des Jugendmedienschutzes: Der neue Jugendmedienstaatsvertrag (Bundesländer der Bundesrepublik Deutschland 2016) enttäuschte viele Erwartungen. Er machte zahlreiche Regelungen im Vollzug noch komplizierter, ohne dass grundlegende Probleme, z. B. im Bereich der internetgestützten Kommunikationsmöglichkeiten, gelöst werden konnten. Wegen der leichten Zugänglichkeit und Verfügbarkeit der Medien schon für kleine Kinder wird der Medienschutz einerseits vielfach für obsolet erklärt. Medienindustrie und Medienanbieter wehren sich gegen alle weitergehenden Auflagen zum Medienschutz und versuchen die Verantwortung für den Medienschutz auf die Medienpädagogik abzuwälzen. Andererseits halten viele Medienpädagoginnen und -pädagogen die Medienkompetenzförderung für einen besseren Kinder- und Jugendmedienschutz, der nach ihrer Meinung die Heranwachsenden zum Selbstschutz befähigt und gesetzliche Einschränkungen weitgehend überflüssig macht (Aufenanger 2008, 61 ff.). Aber die Medienpädagogik sieht sich selbst zunehmend mit der Unkontrollierbarkeit des Medienhandelns der Kinder 
und Jugendlichen konfrontiert und kämpft um eine stärkere Verankerung der Medienbildung als Pflichtaufgabe in den Erziehungs- und Bildungseinrichtungen sowie um eine Verbesserung der personellen und finanziellen Ressourcen für ihre Arbeit (Initiative D21 2016; Niesyto 2011).

Um ein gedeihliches Aufwachsen unserer Kinder und Jugendlichen unter diesen Bedingungen einer mediatisierten Lebenswelt zu gewährleisten, reichen meines Erachtens eine Verbesserung des Medienschutzes und eine Stärkung der Medienpädagogik je für sich nicht aus. Vielmehr müsste - so meine These - die Wirksamkeit von Medienschutz und Medienpädagogik durch ein aufeinander abgestimmtes, soweit als möglich institutionalisiertes Zusammenwirken verbessert werden. Gedeihliches Aufwachsen bedeutet für mich eine vor schwer wiegenden Beeinträchtigungen und Gefährdungen geschützte, durch Erziehung und Bildung geförderte Entwicklung der Persönlichkeit, mit dem Ziel sozialer Integration sowie intellektueller und moralischer Autonomie. Dafür ist es nach meiner Überzeugung erforderlich, den inneren Zusammenhang zwischen Medienschutz und Medienpädagogik zu untersuchen. Deshalb möchte ich im Folgenden klären, welche Faktoren das Medienhandeln der Kinder und Jugendlichen bestimmen und welche Einwirkungsmöglichkeiten sich für Medienschutz und Medienpädagogik durch eine kontextuelle Steuerung ihres Medienhandelns eröffnen. Für ihr abgestimmtes Zusammenwirken auf dieser Basis will ich am Schluss ein praktisches Konzept skizzieren.

\section{Ausgangslage}

Die Notwendigkeit ihres Zusammenwirkens ergibt sich aus der grundlegenden pädagogischen Ambivalenz der Medien, die sowohl zum Nutzen als auch zum Schaden der Heranwachsenden verwendet werden können sowie aus der zunehmenden Unkontrollierbarkeit des Mediennutzungsverhaltens. Bezüglich der Ambivalenz der Medien lassen alle Forschungsergebnisse zum Medienumgang der Heranwachsenden klar erkennen: Sowohl Lern- und Entwicklungschancen der digitalen Medien sowie Kommunikationstechniken als auch Entwicklungsgefährdungen und Schutzbedürfnisse ergeben sich längst nicht mehr allein aus der Nutzung problematischer Medienangebote. Sie resultieren einerseits immer häufiger 
aus völlig neuen und scheinbar unbegrenzten Handlungs- und Kommunikationsmöglichkeiten mit den digitalen Medien, in den Computerspielen und den sozialen Medien. Andrerseits entstehen sie aus neuen Formen eines problematischen Mediennutzungsverhaltens, die als Ergebnis gezielter Manipulationen durch ausgefeilte, von Algorithmen gesteuerte Techniken im Netz angesehen werden müssen (Feierabend u. a. 2020).

Diese vielfältigen Umgangsformen werden von den Heranwachsenden ganz unbekümmert praktiziert, aber das geschieht nie in einem luftleeren Raum: Sie sind eingebettet in die Kontexte ihrer alltäglichen Lebenswelt, in die sozialen Systeme der Familie, Kita, Schule, Peergroup und Freizeit, in denen sie heranwachsen. Diese aber verändern sich selbst rasch und tiefgreifend infolge der fortschreitenden Mediatisierung aller Lebensbereiche durch Digitalisierung, Datafizierung, Algorithmisierung und Vernetzung.

Damit steht der Medienumgang der Kinder und Jugendlichen in den sich wandelnden, mediengeprägten Kontexten ihrer alltäglichen Lebenswelt im Zentrum der Analyse ihrer Entwicklungsbedingungen und bildet den Rahmen für ein Erfolg versprechendes medienpädagogisches Handlungskonzept.

Ein Theorieansatz für eine solche Analyse müsste einerseits die Bedingungen beschreiben, unter denen die Medien neue Lern- und Bildungschancen gewähren und andererseits gefährdende Medienwirkungen, sekundäre Wirkungsdimensionen problematischer Angebote, das Ausmass der Manipulation des Medienhandelns und die Entstehung problematischer Medienhandlungsmuster (z. B. exzessive Mediennutzung) sowie ihre Folgen erklären können. Der Theorieansatz müsste so konstruiert sein, dass er sowohl die Verantwortlichkeit der Medienproduzenten und -anbieter als auch der pädagogisch Handelnden sowie die Eigenverantwortung der Heranwachsenden für die Auswahl und die Folgen ihres Medienhandelns beschreiben könnte. Eine Beschreibung dieser komplexen Zusammenhänge erscheint mir nur möglich, wenn die Bedingungen des Medienhandelns der Kinder und Jugendlichen aus einer systemorientierten Perspektive untersucht werden (Spanhel 2006, 21 ff.). Auf die Leistungsfähigkeit eines systemtheoretischen Ansatzes bei der Analyse von Systemen «organisierter Komplexität» hat bereits Willke (1991, 150 ff.) hingewiesen. Nach meinem Eindruck besitzt er mehr Erklärungskraft für die Frage nach den medialen Bedingungen des Aufwachsens als der neu entwickelte 
Theorieansatz «kommunikative Figurationen» (Hepp und Hasebrink 2014), der sich auf die Frage richtet, welche Transformationen der Konstruktion sozialer Wirklichkeit in diesen Figurationen durch die tiefe Mediatisierung der Gesellschaft ausgelöst werden.

Aus systemtheoretischer Sicht richtet sich der Blick grundsätzlich nicht auf einzelne Phänomene oder Situationen, sondern auf Beziehungen zwischen Systemen. Ich betrachte daher im Folgenden die Kinder und Jugendlichen als autonom handelnde psychische Systeme, die vielfältige Beziehungen zu verschiedensten Medienangeboten als Sinnsysteme aufbauen. Dieses Medienhandeln findet im Kontext der sozialen Systeme ihrer Alltagswelt (Familie, Kita, Schule, Peergroup, Freizeit) statt und beruht auf medialen Kommunikationsprozessen. In diesen Austauschprozessen wirken die sozialen Systeme auf die Heranwachsenden und ihr Medienhandeln zurück. Willke (2005) bezeichnet alle diese sinnverarbeitenden Systeme als symbolische Systeme, weil die medialen Kommunikationen, auf denen sie beruhen, auf dem Gebrauch von Symbolen gründen. (Das alles fundierende Symbolsystem ist die Sprache) Durch eine Analyse der Beziehungsmuster dieser kommunikativen Austauschprozesse möchte ich die Faktoren aufdecken, die das Medienhandeln der Heranwachsenden bestimmen und verdeutlichen, welche Einflussmöglichkeiten Medienschutz und medienpädagogische Massnahmen in diesem Faktorengeflecht haben und wie sie durch ein konzertiertes Zusammenwirken die Bedingungen des Aufwachsens verbessern könnten. Die praktischen Konzepte, die ich im letzten Teil der Untersuchung skizziere, beruhen auf dem Modell einer kontextuellen Steuerung des Medienhandels der Heranwachsenden, als einzige Möglichkeit, auf ein autonomes System einzuwirken (vgl. Willke 2005, 11; 286 ff.) 


\section{Das Medienhandeln der Kinder und Jugendlichen aus systemtheoretischer Perspektive}

\subsection{Medienhandeln als gelingende Kommunikation}

Im Folgenden betrachte ich Kinder und Jugendliche als psychische Systeme, deren Medienhandeln aus dem selbstgesteuerten Zusammenwirken von Wahrnehmungs-, Gefühls-, Denk-, Bewertungs- und Handlungsmustern resultiert. Alle psychischen Systeme erhalten ihren Sinn, ihre Autonomie und ihre Handlungsfähigkeit durch Abgrenzung von ihrer biologischen, dinglichen und sozialen Umwelt (Luhmann 1991, 4:35) Damit sie sich entwickeln können, müssen sie sich gleichzeitig in vielfältige Beziehungen zu Umwelten verwickeln, die für sie bedeutsam sind. Das sind die sozialen SYsteme Familie, Schule, Peergroup sowie die Medienangebote als symbolische Sinnsysteme, die ihnen Kommunikationsofferten anbieten (Willke 2005, 57 ff.). Über Kommunikationsprozesse stehen Kinder und Jugendliche einerseits in vielfältigen Austauschprozessen mit diesen Umwelten, damit sie überhaupt lernen und sich entwickeln können. Um autonom handeln und ihre Identität erhalten zu können, müssen sie sich andererseits aber auch von ihnen abgrenzen und die Differenz zu ihnen über ihre Wahrnehmungs- und Handlungsmuster regulieren (Willke 1991, 37 ff.).

Diese Beziehungen in Form von medialen Kommunikationsprozessen bilden die Grundlage und die sozialen Systeme als relevante Umwelten die Rahmen für die Entwicklung und Sozialisation der Kinder und Jugendlichen. Medien sollen hier mit dem Medienbegriff von Rusch als «konventionalisierte Orientierungsmittel, d. h. als konventionalisierte Kommunikations- und/oder Rezeptionsmittel» (Rusch 2007, 14; kursiv im Org.) gekennzeichnet werden. Diese begriffliche Fassung macht es möglich, die Ebenen des individuellen Handelns und des Sozialen zu integrieren (vgl. Abb. 1). Eine gedeihliche Entwicklung der Heranwachsenden ist nur unter der Voraussetzung möglich, dass es zu einer Verständigung mit den Kommunikationspartnern in den sozialen Systemen kommt. Medienhandeln als Kommunikation aber gelingt nur dann, wenn alle Beteiligten in einem gewissen Umfang über geteiltes Wissen, eine gemeinsame Sprache und Lebenswelt und gemeinsame Konventionen und Normen verfügen. Diese 
Basis für Verständigung müssen die Heranwachsenden im Sozialisationsprozess erst erwerben und in unzähligen Situationen festigen. Wenn sie bestimmte Handlungsziele erreichen wollen, müssen sie erst lernen, wie sie sich mit Hilfe der Medien als «konventionalisierte Kommunikationsund/oder Rezeptionsmittel» mit anderen verständigen können. Nur durch Bezug auf eine gemeinsame Basis wird ihr Medienhandeln zu einem Kommunikationsangebot für andere, das diese verstehen können, dem sie einen bestimmten Sinn zuschreiben und mit ihren eigenen Kommunikationen daran anschliessen können (Rusch 1999, 173 f.). Das heisst aber keineswegs, dass kommunikative Verständigung immer auf Konsens hinausläuft. Beispiele dafür liefern viele konflikthafte Auseinandersetzungen zwischen Eltern und Kindern oder Lehrpersonen sowie Schülerinnen und Schülern. Gerade in pädagogischen Bereichen ist Kommunikation eher Dissensorientiert und bezieht daraus ihre besondere Dynamik und Motivation. Häufig sind diese dissensgesteuerten Kommunikationen in Rahmen eingelagert, in denen ein institutionalisierter Konsens über die Rahmenbedingungen möglichen Dissenses durch Einverständnisse und gemeinsame Überzeugungen abgesichert wird. Am Beispiel der Auseinandersetzungen um eine Verbesserung der Schulqualität sehen wir allerdings, dass ein solcher übergreifender Konsens auch in pädagogischen Einrichtungen immer brüchiger wird.

In Anlehnung an S. J. Schmidt (1998) könnte man dieses geteilte Wissen als gemeinsames «Wirklichkeitsmodell» eines sozialen Systems bezeichnen, gewissermassen als eine «kognitiv, emotional und normativ verbindliche Weltanschauung», die ein soziales System dauerhaft zusammenhält und ihm eine bestimmte Identität verleiht. Allerdings beobachten wir in der modernen segregierten Mediengesellschaft, dass sich die Sozialsysteme, in denen die Kinder und Jugendlichen heranwachsen, in ihren «Wirklichkeitsmodellen» immer stärker voneinander unterscheiden (ebd. 1998, 64). Dann ist die Frage, wie die Heranwachsenden unter diesen heterogenen Bedingungen die notwendige Basis für gelingende Verständigung als Bedingung der Möglichkeit für eine Erfolg versprechende Sozialisation ausbilden, festigen und immer weiter entwickeln können (Spanhel 2013b). Das betrifft insbesondere eine gemeinsame Sprache als das erste und fundamentale Kommunikationsmedium, die samt des in sie eingelagerten 
kollektiven Wissens ebenfalls im Sozialisationsprozess erworben werden muss (Schmidt 2000, 32 ff.). In der Alltagswelt übernehmen die Kinder und Jugendlichen im Rahmen von Familie, Kita, Schule und Peergroup die Sprache, das kollektive Wissen, die gemeinsamen Werte und Normen, Traditionen und Verhaltensmuster des jeweiligen Systems als Basis für gelingende Verständigung.

Alle diese Prozesse sind gleichzeitig in den übergeordneten Rahmen des gesellschaftlichen Mediensystems als symbolisch-kulturelles Sinnsystem eingelagert (vgl. Abbildung 2: Äusserster Rahmen). Dieser umfassende Rahmen kann mit dem Begriff Kultur als Gesamtzusammenhang von Wirklichkeitsmodellen und Kulturprogrammen, als «Sinnmaschine Gesellschaft» (Schmidt 2000, 36) beschrieben werden. Kulturprogramme enthalten seiner Ansicht nach Regeln, Prinzipien und Themen, die aus erfolgreichen Problemlösungen resultieren, die zu ihrer Weiterentwicklung vor allem die Kinder und Jugendlichen als immer neue Programmanwender brauchen. Indem die Heranwachsenden Medien, Software, Plattformen und Medienangebote in den Kontexten ihrer Alltagswelt auf bestimmte Weise nutzen, orientieren sie sich am Wissen, an den Regeln, Normen und den Wirklichkeitsmodellen der Medienkultur. In diesem Rahmen vollzieht sich ihre Entwicklung als Konstruktion und Transformation ihrer inneren Strukturen (Spanhel 2010c). Gleichzeitig tragen sie mit ihrer Teilhabe an den medialen Kommunikationsprozessen zur Stabilisierung und Weiterentwicklung sowohl der sozialen Systeme als auch des Mediensystems und der Kulturprogramme bei. «Dieser Doppelaspekt erlaubt die Beobachtung, dass der Mensch Schöpfer aller Kultur ist und zugleich die Menschen Geschöpfe einer je spezifischen Kultur sind.» (Schmidt 2000, 36, kursiv im Org.) Kulturprogramme reduzieren die unvorstellbare Komplexität der prinzipiell möglichen Kommunikationsformen in der Gesellschaft, sichern damit ihren Fortbestand und stiften zugleich individuelle und soziale Identität. Mit ihrer Weitergabe im Sozialisationsprozess sind für die Heranwachsenden Verpflichtungen verbunden, die die Beziehungen zwischen den sozialen Ordnungen und der individuellen Freiheit kontrollieren. Diese Kontrolle folgt jedoch nicht dem kausalen Verursacherprinzip, sondern durch «kulturell programmierte Bedeutungen», wie sie insbesondere in der Sprache zum Ausdruck kommen: 
«Sprachliche Sozialisation ist besonders rigide, und die Regeln gesellschaftlich korrekter Anwendung sprachlicher Mittel kondensieren gesellschaftlich akzeptierte Erfahrungen, Affekte und Überzeugungen.» (Schmidt 2000, 37; zu diesen Leistungen der Sprache vgl. auch Willke 2005, 35).

Von gelingender Kommunikation soll dann die Rede sein, wenn sie entsprechend dem beschriebenen Doppelaspekt sowohl die Entwicklung der Heranwachsenden als auch die des jeweiligen sozialen Systems durch immer weitere Anschlusskommunikationen fördert (Rusch 1999, 174). Aber wie ist das überhaupt möglich, obwohl doch psychische und soziale Systeme autonom nach dem Prinzip der Selbstorganisation operieren und von aussen nicht direkt beeinflussbar sind? Am Beispiel der Familie lässt sich beobachten, wie Systeme durch vielfältigen Austausch von Kommunikationen im jeweils anderen System Wirkungen auslösen können: Je enger die Beziehung der Kinder zur Familie, desto intensiver verlaufen die Kommunikationsprozesse in dieser Gemeinschaft ab. Dabei stellt sich eine besondere Form der Beziehung zwischen den einzelnen Familienmitgliedern und dem System Familie her: Die autonom agierenden Systeme sind über die Kommunikationsprozesse aneinander gekoppelt. Sie regen sich wechselseitig zu ähnlichen Konstruktionen von Wirklichkeit an. Je enger die Beziehungen auf der Grundlage medialer Kommunikationen, desto stärker werden sich die Systeme in ihren Strukturen einander angleichen (Zum Begriff der strukturellen Kopplung Rusch 1999, 160 ff.) Dabei kommt es zwischen den Familienmitgliedern, aber auch zwischen den Mitgliedern und der Familie als System zu parallel ablaufenden, sich gegenseitig bedingenden Entwicklungsprozessen, die als Ko-Ontogenese bezeichnet werden (Büeler 1994, 105 ff.; 205). Sie beruhen auf der Ausrichtung des Handelns an gemeinsamen Sinnorientierungen. Das ist die Grundlage für die Sozialisationsprozesse, die aber heute immer brüchiger wird (Kammerl und Kramer 2016). 


\subsection{Medienhandeln als Ergebnis von Prozessen der Selbstregulation}

Sinnorientierungen steuern die Kommunikationsprozesse und das Medienhandeln. Sie steuern die Auswahl einer Kommunikationsofferte aus der Komplexität der Sinnorientierungsmöglichkeiten, die das Mediensystem anbietet (siehe Abbildung 1: Selektion 1). Die Eindrücke des gewählten Medienangebots, z. B. eines gewalthaltigen Computerspiels, stossen strukturelle Transformationen in den individuellen Wahrnehmungs-, Gefühls-, Wertungs- und Denkmustern der Heranwachsenden an, die selbstreguliert ablaufen. Das Ergebnis dieser Regelungsprozesse besteht zum einen darin, dass dem Sinnangebot eine individuelle Bedeutung zugeschrieben und zum anderen aus einer Vielzahl von Handlungsmöglichkeiten eine Anschlusshandlung zur Sinnverwirklichung ausgewählt wird (Selektion 2). Dieses Medienhandeln ist von den Kommunikationspartnern beobachtbar und stellt für sie wiederum ein Kommunikationsangebot dar, an das weitere, jedoch nicht vorhersehbare Kommunikationen anschliessen können. Sinn fungiert als Steuerungskriterium für das Medienhandeln, aber dieses wird durch das Medienangebot nur ausgelöst. Es resultiert allein aus den autonomen Operationen des psychischen Systems. Die Auswahl aus der Komplexität der Sinnorientierungsmöglichkeiten wird als Selektionszwang, die Wahl einer Handlung aus den Möglichkeiten zur Sinnverwirklichung (Kontingenz) wird als Handlungsfreiheit erlebt. Das erklärt, warum ein Medienangebot nicht direkt auf die Kinder und Jugendlichen einwirken und ihr Medienhandeln keineswegs bestimmen kann. Es lässt sich daher nicht von vorneherein sagen, auf welche Weise es die Entwicklung der Heranwachsenden fördert, beeinträchtigt oder gefährdet (Spanhel 2006, 32 ff.). 


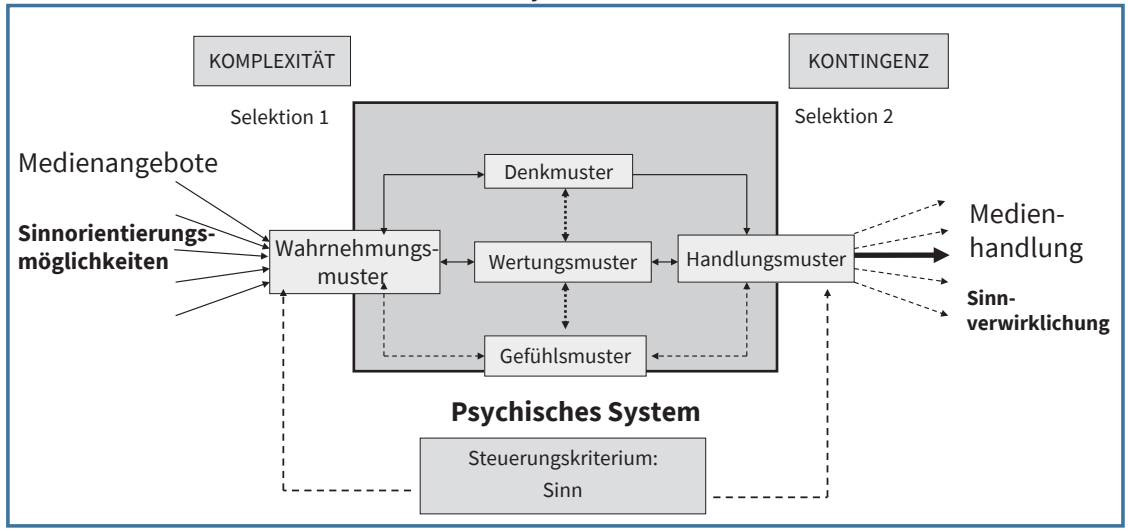

Abb. 1.: Medienhandeln (eigene Darstellung, in Anlehnung an Willke 1991, 28).

Wie Abbildung 1 zeigt, ist das Medienhandeln Ergebnis von Regelungsprozessen zwischen sozialen und psychischen Systemen auf der Grundlage des Steuerungskriteriums Sinn (Willke 1991, 151). Dazu ein Beispiel: Die Computerspielindustrie (soziales System als Rahmen) bietet den Jugendlichen eine Fülle an verlockenden Sinnorientierungsmöglichkeiten in Form spannender und gewalthaltiger Videospiele. Verstärkt durch aggressive Werbung und bedrängt von seinen Freunden wählt ein Jugendlicher aus dieser Komplexität ein bestimmtes gewalthaltiges Spiel aus (Selektion 1). Diese Entscheidung löst im Zusammenspiel seiner individuellen Wahrnehmungs-, Denk-, Gefühls- und Wertungsmuster strukturelle Transformationen in seinem psychischen System aus. Als Ergebnis dieser inneren Regulationen schreibt der Jugendliche dem Sinnangebot eine individuelle Bedeutung zu: Er sieht darin die Möglichkeit, seinen Ehrgeiz zu befriedigen und die Anerkennung seiner Freunde zu gewinnen. Er wählt daher aus einer Vielzahl von Handlungsmöglichkeiten im Spiel (Kontingenz) die Form eines verbissenen Kampfes um höhere Wertungspunkte gegen den Computer aus (Selektion 2). Im folgenden Medienhandeln vollzieht er seine persönliche Sinnverwirklichung, erlebt seine Geschicklichkeit und seinen Spielerfolg, was verstärkend auf dieses Handlungsmuster zurückwirkt.

Seine Freunde beobachten sein ehrgeiziges Spiel (kommentieren, belächeln, stören es) und bewundern (beneiden oder missgönnen ihm) den Erfolg. Diese Beobachtungen wirken auf die Peergroup zurück, sie stabilisieren und verstärken in der Gruppe ein bestimmtes Selektionsmuster 
(Gewaltspiele) sowie ein Erfolg versprechendes Medienhandlungsmuster zur Befriedigung bestimmter Bedürfnisse und Wünsche (Selbstwirksamkeit, Anerkennung). Wenn sich die Jugendlichen begeistert über ihre Spielerlebnisse austauschen, könnten auch andere zu diesem Spiel animiert und dadurch der Zusammenhalt der Gruppe gefestigt werden. Auf diese Weise kommt es durch strukturelle Kopplung zwischen den einzelnen Jugendlichen und der Gruppe zur Ausbildung gemeinsamer Sinnorientierungen beim Computerspiel. So kann sich um ein bestimmtes Computerspiel (z. B. WoW) ein Fanclub organisieren.

\subsection{Sinnorientierungen als Steuerungsfaktor für Medienhandeln}

Sinn ist das Steuerungskriterium für alle psychischen und sozialen Systeme (Büeler 1994, 114 f.). Das Medienhandeln der Heranwachsenden beruht auf der grundsätzlichen menschlichen Fähigkeit, sich sinnhaft in der Welt zu orientieren. Das gibt Menschen die Möglichkeit,

«[...] über Sprache und andere Sinnsysteme (z. B. symbolische Sinnsysteme der Medien; D.S.) soziale Beziehungen aufzubauen und Herausbildung und Abgrenzung sozialer Identitäten nach sinnhaften Kriterien zu steuern." (Willke 1991, 29)

Sich sinnhaft in der Medienwelt zu orientieren heisst, aus den Sinnorientierungen der Medienangebote eine Orientierung auszuwählen und im Medienhandeln zu verwirklichen. (Zur Bedeutsamkeit der Unterscheidung von Sinnorientierung als Ausrichtung von Handlungen und Handlung als Realisierung von Sinnorientierungen S. J. Schmidt 2004, 139).

Sinn ergibt sich aus Verweisungszusammenhängen und der damit verbundenen Begrenzung von Handlungsmöglichkeiten durch Normen, Regeln oder Routinen des Mediensystems. Der Sinn von Grenzen ist die Begrenzung von Sinn (Willke 1991, 42). Solche Grenzsetzungen werden in Form von Handlungsrahmen oder Kontexten in der Alltagswelt markiert (Spanhel 1999b). Dabei ist zwischen den von den sozialen Systemen vorgegebenen Sinnorientierungsangeboten und den Bedeutungen zu unterscheiden, die Kinder und Jugendliche selbst ihrem Medienhandeln geben. Die 
subjektiven Bedeutungen, die sie den Medienangeboten zuschreiben, erfolgen immer unter den situativen Bedingungen eines begrenzten alltagsweltlichen Handlungsrahmens: «Der Kontext bestimmt die Bedeutung eines Mediums» (Bateson 1990). Erst durch die Kontexte in Wechselwirkung mit den Person-internen Handlungsbedingungen (insbesondere der individuellen Wertrangordnung) werden die Medienhandlungen für die Kinder und Jugendlichen in spezifischer Weise bedeutsam, z. B. wenn sie ihnen für die Lösung eines akuten Alltagsproblems oder im grösseren Zusammenhang für die Bewältigung einer Entwicklungsaufgabe hilfreich erscheinen (Spanhel 2006, 88 ff.). Diese subjektive Bedeutungszuweisung ist das entscheidende Kriterium bei der Auswahl aus den Sinnorientierungsangeboten der Medien.

Das handelnde Subjekt bestimmt den Kontext. Es macht eben einen grossen Unterschied, ob Jugendliche ein gewalthaltiges Computerspiel im Kontext der Peergroup mit ihren Freunden spielen und sich dabei als cool und spielkompetent darstellen, um grössere Anerkennung in der Gruppe zu bekommen oder ob sie dieses Spiel im familiären Kontext vor den Eltern als harmlos verteidigen, um es nicht löschen zu müssen und im Spiel weiterhin ihre angestauten Aggressionen abbauen und ihr Bedürfnis nach Angst-Lust-Erlebnissen befriedigen zu können. Im ersten Fall geht es um einen Regelungsprozess zwischen den Normen der Peergroup einerseits (sozial vorgegebene Sinnorientierung) und dem wichtigen Anliegen der Jugendlichen andererseits, durch Anerkennung in der Gruppe soziale Zugehörigkeit zu erfahren und die Stabilisierung ihrer Identität zu sichern (subjektive Sinnorientierung). Im zweiten Fall suchen die Jugendlichen eine Regelung zwischen dem Verbot der Eltern (soziale Orientierung) und ihren inneren Triebkonflikten (subjektive Orientierung) herzustellen. In beiden Fällen können wir aus der Beobachterperspektive ihrem $\mathrm{Me}$ dienhandeln eine bestimmte subjektive Bedeutung durch den Bezug auf die Bewältigung einer Entwicklungsaufgabe zuschreiben, sei es die Eingliederung in eine Peergroup, sei es die eigenverantwortliche Regelung von Bedürfnissen oder die Ablösung von der elterlichen Bevormundung. Das Medienhandeln ist immer in soziale Kontexte eingebettet, die für den Erhalt der Identität der Jugendlichen bedeutsam sind. Deshalb müssen sie in ihrem Handeln auch die Erwartungen der jeweiligen sozialen Systeme 
berücksichtigen. Die Frage ist, wie die sozialen Systeme als Kontexte so pädagogisch gestaltet werden können, dass es den Heranwachsenden gelingt, ihre Bedürfnisse, Wünsche und Interessen im Medienhandeln mit den begründeten Erwartungen des sozialen Systems so abzustimmen, dass sich das Medienhandeln förderlich auf ihren Entwicklungs- und Bildungsprozess auswirkt.

\section{Sinn stiftende Kontexte für das Medienhandeln der Heranwachsenden}

Als Fazit aus den bisherigen Überlegungen ist festzuhalten: Es sind die alltagsweltlichen Kontexte (im Sinne von Bateson 1990, 25ff.; 150ff.) oder auch Rahmen (im Sinne von Goffman 1989, 31ff.), die dem Medienhandeln der Heranwachsenden ein begrenztes Angebot an Sinnorientierungen vorgeben. Daraus wählen sie eine Sinnorientierung aus, die für sie eine besondere Bedeutung hat und die sie dann in ihrem Medienhandeln verwirklichen. Im Folgenden möchte ich versuchen, die Komplexität dieser ineinander verschachtelten Kontexte zu entwirren und ihre Sinn stiftenden Funktionen für das Medienhandeln der Heranwachsenden zu beschreiben (Abbildung 2). Vor diesem Hintergrund werden die Notwendigkeit und die Möglichkeiten eines Zusammenwirkens von Kinder- und Jugendmedienschutz und Medienpädagogik auf der Basis einer kontextuellen Steuerung ihres Medienhandelns erkennbar. 


\section{Mediatisierte Lebenswelt}

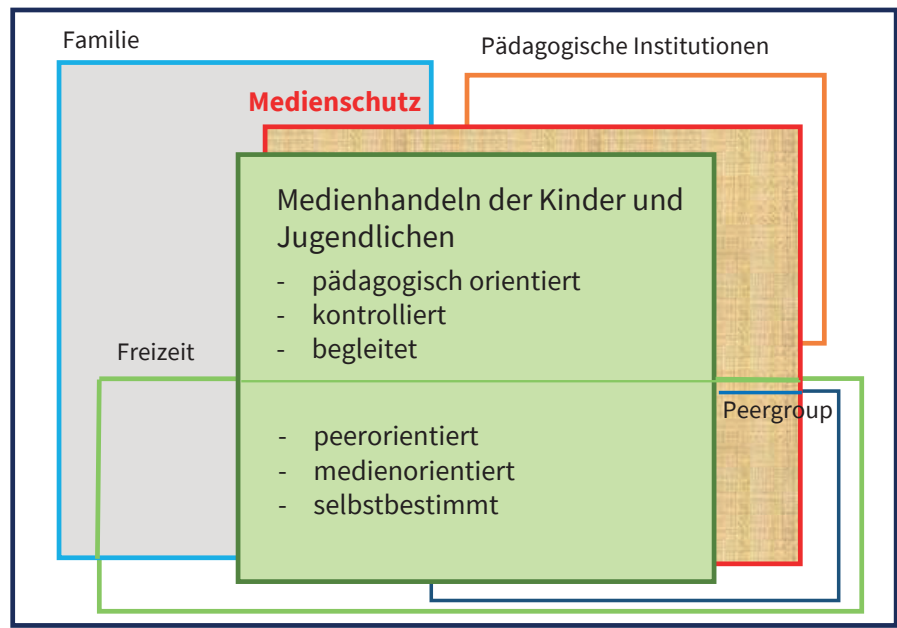

Sinn stiftende Kontexte

Abb. 2.: Verschachtelung der Sinn stiftenden Kontexte (eigene Darstellung).

\subsection{Die Medienkultur als umfassender Kontext}

Den äussersten, alles umfassenden Rahmen für das Medienhandeln der Heranwachsenden stellt die mediatisierte Lebenswelt dar. Mediatisierte Lebenswelten (Krotz 2001, 29 f.) beruhen auf einem integrierten Kommunikationssystem, das alle kulturellen Sinngehalte und Ausdrucksweisen in einer

«Kultur der realen Virtualität» vereint (Castells 2001, 425). Das bedeutet, dass die Texte der digitalen Medien die ganze menschliche Erfahrung absorbieren und alle Wirklichkeiten durch Symbole kommuniziert werden. Kultur ist daher in der heutigen Gesellschaft immer Medienkultur. Sie bildet den umfassendsten medialen Kontext, der auch die medialen Kontexte aller anderen symbolischen und sozialen Systeme der Alltagswelt strukturiert. Die Kinder und Jugendlichen haben über die digitalen Medien weitgehend ungehinderten Zugang zu dieser Medienkultur in der ganzen Breite und Vielfalt ihrer Ausprägungen. Die Sinnorientierungen, die sie daraus auswählen und in ihrem Medienhandeln verwirklichen, bilden und stärken die Gemeinsamkeiten im alltäglichen Zusammenleben und sind aus systemorientierter Sicht der «Kitt», der sie durch strukturelle Kopplung an die sozialen Systeme der Gesellschaft bindet. 
«Kommunikation (in der Form des Medienhandelns; D. S.) bringt Individuen und soziale Systeme auf der gemeinsamen Basis von Sinn in eine Relation struktureller Kopplung, die Zusammenhang und Distanz, Autonomie und Relationierung zugleich erlaubt und fordert." (Willke 2005, 18)

War es früher das Medium der Sprache, so ist es heute darüber hinaus der Gebrauch der digitalen, insbesondere der Onlinemedien, der der nachfolgenden Generation die Teilhabe am sozialen Zusammenleben und den Errungenschaften der Kultur gewährt und damit die Erhaltung und Weiterentwicklung von Gesellschaft und Kultur garantiert. Daneben darf allerdings die Bedeutung der «symbolisch generalisierten Medien» und ihre Wirksamkeit in der heutigen Gesellschaft nicht unterschätzt werden (Thye 2013, 175; Luhmann 1991; Spanhel 2013b).

Aus dieser Sicht sind Heranwachsende und Mediensystem in ihrer Beziehung mehr denn je über das Medienhandeln zeitlich und räumlich sehr eng aneinander gekoppelt und fungieren jeweils gegenseitig füreinander als bedeutsame Umwelten. Eine Beziehung ist aus systemischer Sicht «immer Produkt einer doppelten Beschreibung» (Bateson 1990, 165). Deshalb muss die Beziehung zwischen Heranwachsenden und den Medienkulturen sowohl aus der Sicht der Heranwachsenden als auch aus der Perspektive des Mediensystems betrachtet werden.

Für die Heranwachsenden ist das Mediensystem eine relevante Umwelt und von essentieller Bedeutung für ihren Entwicklungsprozess: Es bietet eine Fülle an entwicklungsnotwendigen Handlungsräumen für Welt- und Selbsterfahrung und für soziale Teilhabe. Die digitalen Medien eröffnen vielfältige, offene Handlungsräume, in denen die Heranwachsenden relativ frei von pädagogischen Kontrollen ihre je nach Alter neu erworbenen Wahrnehmungs-, Gefühls-, Wertungs-, Denk- und Handlungsmuster erproben, festigen und ausdifferenzieren können. Diese Erfahrungsräume gewähren ihnen Partizipation an allen Lebensbereichen, ermöglichen eine Befriedigung ihrer Grundbedürfnisse nach Selbstwirksamkeit, Anerkennung, Autonomie und Selbstdarstellung und unterstützen die selbständige Bewältigung der Entwicklungsaufgaben (Spanhel 2013a). Andererseits erwachsen aus der Offenheit und Unkontrollierbarkeit, aus der 
Widersprüchlichkeit und Unpersönlichkeit der medialen Erfahrungsräume und Handlungsmöglichkeiten neue Probleme und Entwicklungsgefährdungen. Und die Möglichkeiten zu einer eigensinnigen Nutzung der Medienangebote führen zu einer Ausdifferenzierung der Sozialisationsprozesse (Fleischer 2018, $287 \mathrm{ff}$.).

Hinsichtlich der zeitlichen Dimension beschleunigen die Beziehungen zu den Medienkulturen einerseits die Lernfähigkeit und die kognitive Entwicklung der Heranwachsenden (Spanhel 2005, 31 f.). Andererseits werden sie in den sensiblen Phasen ihrer Entwicklung besonderen Gefährdungen ausgesetzt, denn sie können sich der Faszination der Medien kaum entziehen. Das belegen schon die neuesten Forschungsergebnisse über das zeitliche Ausmass der Mediennutzung (Feierabend, Plankenhorn, und Rathgeb 2017). Deren mediale Reize aber bedienen im Übermass jene unbewussten Triebe, Bedürfnisse und Sehnsüchte, die die Heranwachsenden gerade zu beherrschen lernen (Doelker 2002, 105 ff.). Dadurch können der Aufbau stabiler innerer Verhaltenskontrollen bei Kindern und die Entwicklung eines Wertsystems mit einer eigenen Präferenzordnung als Kern der Identität bei Jugendlichen empfindlich gestört werden (Spanhel 2013a).

Für das Mediensystem, für Medienindustrie und Medienanbieter sind die Heranwachsenden eine relevante Umwelt als wichtige Zielgruppe für die Akzeptanz, Erprobung und Platzierung neuer Medienprodukte, Programme und Dienstleistungen im Alltag: Sie sind offen für Produktinnovationen, begierig auf neue inhaltliche Angebote und erproben ohne Vorbehalte auf spielerische Weise die Handlungsmöglichkeiten neuer Produkte. Deshalb kämpft die Medienindustrie darum, Beschränkungen in den Zugangs- und Nutzungsmöglichkeiten der Jugendlichen zu den Medien soweit irgend möglich zu vermeiden. Gleichzeitig dient dieser Kampf um die Aufmerksamkeit und Zuwendung der Heranwachsenden der Gewinnmaximierung.

Aus zeitlicher Sicht sind die Heranwachsenden relevante Umwelt für das Mediensystem, weil sie durch die spielerische Erkundung und Erprobung neuer Anwendungsmöglichkeiten die permanente technische und ökonomische Weiterentwicklung der Medien und Medienangebote vorantreiben und so zur Beschleunigung der Medienentwicklungen beitragen. Damit werden die Heranwachsenden als Konsumenten für die $\mathrm{Me}$ dienanbieter überall und zu jeder Zeit beobachtbar und für die Erfassung 
personenbezogener Daten und ihre Vernetzung sowie für gezielte Werbeund Konsumangebote verfügbar (Feierabend, Rathgeb, und Reutter 2019).

Technisch gesehen beruht die neue Medienkultur auf einer algorithmisch gesteuerten Erzeugung, Verbreitung und Präsentation der $\mathrm{Me}$ dieninhalte und medialen Kommunikationsprozesse. Von herausragender Bedeutung für den Entwicklungsprozess der Heranwachsenden ist die Tatsache, dass die digitalen Medien zur sozialen, sachlichen und kontextuellen Entkopplung des Zeichengebrauchs und dadurch zu einer enormen Zunahme an Kommunikationsmöglichkeiten führen (Thye 2013, 191ff.; 213; Esposito 2010). Diese Entkopplungen bieten für die Kommunikation einen unvorhergesehenen Raum des Experimentierens und der Unwahrscheinlichkeit, einen «Überschuss an Kommunikationsmöglichkeiten» (Luhmann 1996, 11). Sie ermöglichen es, dass in den sozialen Netzwerken heute jeder zum Medienproduzenten werden kann und sie erlauben den Adressaten jede Freiheit im Umgang mit einer Mitteilung. In der Folge dieser Entkopplungen tauchen die heute vielfach beklagten Kommunikationsformen im Netz auf, wie z. B. Hassbotschaften, Drohungen oder Fake News, aus den Tiefenschichten der Person kommende, enthemmte, nicht rational kontrollierte Kommunikationen, aber auch gezielt auf andere Personen oder Gruppen gerichtete Botschaften, die herabsetzen, bedrohen, beleidigen oder verletzen. Damit vervielfachen sie die «Macht des Wortes» in ihren Wirkungsdimensionen. Technisch vermittelte Kommunikation kann sich von der Alltagsrealität abkoppeln und eigene virtuelle Realitäten hervorbringen (Esposito 2010). Dafür steht die ausufernde Computerspielindustrie.

Diese virtuellen Welten zeichnen sich durch eine enorme Zunahme an Beobachtungsmöglichkeiten zweiter Ordnung aus. Die Heranwachsenden können hier Personen und Geschehnisse mit einer im Alltag sonst nicht möglichen Durchsichtigkeit und Notwendigkeit beobachten (Esposito 2010, 166). Damit ist eine unglaubliche Steigerung der Kontingenz, d. h. der Möglichkeiten der Bedeutungszuschreibung und der Freiheit im Medienhandeln verbunden. Das bedeutet grosse Unsicherheiten im Sozialisationsprozess, weil sich die eindeutige Welt einer/eines Heranwachsenden in der Mehrheit der Welten der verschiedenen Beobachter vervielfältigt und jede Beziehung in der eigenen Welt in der Welt eines anderen Beobachters 
anders aussehen kann. Die Heranwachsenden nehmen ihre Alltagswelt immer häufiger aus der Perspektive ihrer medialen Beobachtungserfahrungen wahr. Für ihre Entwicklung stellen diese Beobachtbarkeit und Kontingenzerfahrungen eine besondere Herausforderung dar, weil diese Prozesse der Entkopplung die Orientierungsfunktion der Medien und die oben dargestellten Voraussetzungen für gelingende Sozialisation untergraben. Sie stellen aber auch die pädagogisch Verantwortlichen vor die schwierige Aufgabe einer Beobachtung dieser Beobachtungserfahrungen, wenn sie die Heranwachsenden bei der Bewältigung medienbedingter Entwicklungsprobleme unterstützen wollen (Fleischer 2018, 292).

Die Beziehung der Heranwachsenden zu der digitalen Medienkultur in der besonderen Form der medialen bzw. kommunikativen Kopplung ist die unabweisbare Bedingung ihres Aufwachsens in der heutigen Gesellschaft und umfasst alle Möglichkeiten und Chancen, aber auch alle Gefährdungen und Beeinträchtigungen eines gedeihlichen Aufwachsens. Im Folgenden ist genauer zu untersuchen, wie und mit welchen Konsequenzen sich diese Kopplung in den alltäglichen Lebensräumen mit ihren unterschiedlichen medialen Kontexten auf den Entwicklungsprozess der Kinder und Jugendlichen auswirkt.

\subsection{Gesetzgeberische Kontexte}

In ihrer mediatisierten Alltagswelt (Hartmann und Hepp 2010) spielt sich das Medienhandeln der Kinder und Jugendlichen innerhalb des gesetzlich vorgegebenen Rahmens des Kinder- und Jugendmedienschutzes ab, auch wenn ihnen das in den meisten Fällen gar nicht bewusst ist. (Auf die gesetzlichen Grundlagen und die einzelnen Institutionen des Kindere- und Jugendmedienschutzes und ihre Arbeitsweise kann hier nicht näher eingegangen werden). Dieser gesetzliche Rahmen schränkt die Sinnorientierungsmöglichkeiten in allen medialen Handlungskontexten ein. Damit sollen von vorneherein all jene Medienangebote und Handlungsmöglichkeiten ausgeschlossen werden, die die Entwicklung der Heranwachsenden gefährden und die Würde ihrer Person verletzen. Zugleich geht es darum, ihr Medienhandeln an den Normen und Werten der Gesellschaft auszurichten. In diesem Sinn ist der Jugendmedienschutz eine Staatsaufgabe (Baum 2008) und dafür werden die Medienproduzenten und -anbieter in 
die Verantwortung genommen. Diese Einschränkung der Sinnorientierungsmöglichkeiten dient nicht nur zum Schutz der Heranwachsenden, sondern zugleich zu ihrer Sozialisation und damit ist der Medienschutz mit seinen Einrichtungen und Massnahmen eine genuin pädagogische Institution.

\subsection{Alltagsweltliche Kontexte}

Innerhalb dieser beiden weiten Rahmen ist das Medienhandeln der $\mathrm{He}$ ranwachsenden in verschiedene, pädagogisch gestaltete oder begleitete Handlungsrahmen eingebettet (Abbildung 2): Dies sind in erster Linie die Familie, dann die Erziehungs- und Bildungsinstitutionen (Kindertagesstätten, Schulen) und andere pädagogische Einrichtungen (Heime, Horte, Jugendzentren, Berufsausbildung). Diese Einrichtungen schreiben den Medienangeboten für die Entwicklung der Heranwachsenden besondere Bedeutungen zu und wollen dadurch ihr Medienhandeln an pädagogisch begründeten Sinnorientierungen ausrichten. In der Familie ist das Medienhandeln primär an Sinnorientierungen ausgerichtet, die durch Lebensstil, Kommunikationsmuster und gemeinsame Aktivitäten der Familienmitglieder zum Ausdruck kommen. Es ist in die alltäglichen Lebensvollzüge und Handlungsmuster eingebettet, nach denen das familiäre Zusammenleben organisiert ist (Feierabend, Plankenhorn, und Rathgeb 2017). Als Antwort auf die Kommunikationsangebote der Familie konstruieren die Heranwachsenden ihre eigenen Medienhandlungsmuster, die ihren Alltag strukturieren, aber auch auf das familiäre Zusammenleben zurückwirken. Viele Eltern wollen das Medienhandeln ihrer Kinder pädagogisch begleiten und steuern. Dementsprechend bilden sich in den Familien unterschiedliche Medienerziehungskonzepte aus (Wagner und Gebel 2015; Wagner u. a. 2013).

In der Gesamtheit dieser medialen Kommunikationsprozesse wird ein familientypisches Wirklichkeitsmodell aus geteiltem Wissen und gemeinsamen Normen, Konventionen und Wertorientierungen aller Familienmitglieder etabliert, das den Medien spezifische Bedeutungen zuweist. In diesen Prozessen wechseln sich Phasen der Veränderung und der Stabilisierung in der Entwicklung eines familientypischen Mediengebrauchs 
ab. Unausweichlich kommt es dabei häufig zu Konflikten zwischen Eltern und Kindern hinsichtlich der Bedeutungszuweisung zu einzelnen Medienhandlungsmustern. Sie können fruchtbare Anstösse sowohl für die Entwicklung der Familie als auch der Kinder geben, vor allem, wenn sie diese Probleme thematisieren, darüber streiten und wenn ihnen dabei mehr und mehr die Medialität ihres Handelns bewusst wird.

In den Erziehungs- und Bildungseinrichtungen (Kindertagesstätten, Schulen, Horten) ist das Medienhandeln pädagogisch orientiert, kontrolliert und begleitet. Das bedeutet, dass die Sinnorientierungsmöglichkeiten für das Medienhandeln an übergeordneten Lern- und Bildungszielen ausgerichtet sind. Dafür wurden unterschiedliche Medienbildungskonzepte entwickelt (Schill 2008; Spanhel 1999a; 2006; Tulodziecki und Herzig 2002; Tulodziecki, Herzig, und Grafe 2010). Sie werden häufig immer noch sehr unzureichend umgesetzt (vgl. Initiative D21 2016; Niesyto 2011). Aber durch die Ergänzung der traditionellen Bildungsmedien Sprache und Buch um die digitalen Medien bieten sich den Heranwachsenden vielfältige neue Möglichkeiten zum Lernen mit Medien und zum Aufbau alternativer entwicklungsfördernder Medienhandlungsmuster (Schulz-Zander 2005; Weidenmann 2006; Eickelmann 2010). Die neue Initiative der KMK mit dem Ziel einer flächendeckenden Vermittlung digitaler Bildung (KMK 2016) ist mit einer grossen Euphorie bezüglich einer Verbesserung der schulischen Lernprozesse verbunden, die aber durch die Erfahrungen mit dem HomeSchooling während der Corona-Krise deutlich gedämpft wurde.

Von besonderer Bedeutung für Kinder und Jugendliche sind die Handlungsrahmen in der Freizeit, weil sie dort ihr Medienhandeln weitgehend selbstbestimmt gestalten können. Dabei kommen ihnen ihre Medienausstattung und die unbegrenzten digitalen Erfahrungs-, Lern-, Kommunikations- und Handlungsmöglichkeiten zugute (Spanhel 2020). Mehr denn je spielt sich diese Freizeit in den virtuellen Welten der Medien, des Internet, des Web 2.0, der Online-Videospiele oder in den globalen Netzwerken der Social Media ab. In diesen Medienwelten können sie aus einer riesigen Vielfalt an Sinnorientierungen und Handlungsmöglichkeiten auswählen. Dies geschieht einerseits selbstbestimmt nach individuellen Präferenzen, andererseits häufig nach den Sinnorientierungen einer Peergroup, die mit zunehmendem Alter eine bedeutsame Rolle spielt. Und die Handlungsfreiräume 
der Medien werden umso wichtiger, je mehr sich die Heranwachsenden in ihrer Entwicklung von Eltern sowie Pädagoginnen und Pädagogen ablösen müssen, um ihre eigene Identität aufbauen zu können (Spanhel 2013a). Diese Sinnorientierungen werden durch empirische Untersuchungen bestätigt, die das Medienhandeln in der Freizeit als selbstbestimmt, peerorientiert und medienorientiert ausweisen (Spanhel 1990).

\subsection{Selbstkonstruierte mediale Handlungsrahmen}

Nicht nur in ihrer Freizeit, sondern wo immer Kinder und Jugendliche sich von sozialen oder pädagogischen Kontrollen befreien können, nutzen sie Medien, um eigene Handlungsrahmen zu errichten, die ihre Alltagssituation strukturieren und ihr individuelles oder soziales Handeln organisieren. Das zentrale Merkmal der multifunktionalen Medien, Apps, Plattformen oder Netzwerke ist ihre Offenheit, die den Heranwachsenden als ein «Spielraum der Freiheit» willkommen ist. Sie bieten ihnen - wie empirische Ergebnisse der jährlichen KIM- und JIM-Studien (vgl. Feierabend, Rathgeb, und Reutter 2019; 2020) zeigen - vielfältige Sinnorientierungsangebote, die auf Kommunikation (soziale Netzwerke, Online-Communities, z. B. Facebook, WhatsApp), Information (YouTube, Wikipedia, Suchmaschinen, Open Source Software), Unterhaltung (fiktionale Angebote der Massenmedien, vielfach über das Internet genutzt) und Spiele (Online- und Offlinespiele auf Playstation, Smartphone, Tablet oder Computer) ausgerichtet sind.

Probleme für das Aufwachsen ergeben sich, weil mit der Offenheit der digitalen Medien sowohl Chancen als auch Gefährdungen verbunden sind: Die Chancen liegen in den Handlungsfreiräumen, in denen die Heranwachsenden ihre Kräfte und Fähigkeiten, Gefühle und Interessen experimentierend erproben, ihre Wünsche ausleben und ihre Grundbedürfnisse befriedigen können, was ihnen bei der selbständigen Bewältigung ihrer Entwicklungsaufgaben hilft (Fleischer 2018). Sie können vielfältige Lernund Bildungsprozesse initiieren: Im Rahmen der Online Communities, der Nutzung von sozialen Medien und Plattformen im Netz lernen die Heranwachsenden, sozial und kulturell vielfältige sozio-technische Praktiken (Koenig 2011, 41 ff.) auszubilden. Sie erproben vielfältige Möglichkeiten zur 
Kontaktherstellung und Partizipation, zur Bildung sozialer Gemeinschaften und Entwicklung neuer Kommunikationsformen innerhalb der Regeln und Grenzen, die von Hard- und Software bestimmt werden (z. B. in den Computerspielen: Ruckdeschel 2015). Sie lernen, sich in den wechselnden Beziehungen stets neu zu verorten, ihr Handeln zu reflektieren und aus unterschiedlichen Perspektiven zu beurteilen. Dieses Lernen in offenen medialen Handlungsräumen ist situiert, ein Lernen vom Kontext (nach Bateson Lernen I), spielerisch, motiviert und selbstgesteuert (Spanhel 2020; Theunert 2005). Es beruht auf Erzählen, Nachfragen bei Problemen, Ausprobieren und Experimentieren, Reflektieren und Kommentieren, auf Unterstützung durch Peers und Experten. Es ist vielfach ein kollaboratives Lernen «just in time» im Vollzug der Lösung gerade anstehender eigener Probleme und bei der Verfolgung gemeinsamer Interessen oder Ziele in sozialen Kontexten (Ala-Mutka 2009). In diesem Zusammenhang habe ich von medialen Bildungsräumen gesprochen (Spanhel 2010a; 2010b; 2017).

Aber diese medialen Spielräume bergen auch latente Gefährdungen für den Entwicklungsprozess. Kinder und Jugendliche können als Produzenten, Anbieter oder Verbreiter von Medieninhalten in diesen Handlungskontexten sehr oft mögliche weitreichende Konsequenzen ihrer Aktivitäten für sich selbst, für andere Heranwachsende und für ihre Alltagswelt nicht klar erkennen und abschätzen. Das liegt nicht nur an fehlender Medienkompetenz, sondern an den komplexen Strukturen der Medienangebote und des Netzes, die mögliche Handlungsfolgen verschleiern. Hinzu kommen Gefährdungen durch Formen exzessiver Mediennutzung, Internet- und Computerspielsucht, die schon seit Jahren und häufig sehr kontrovers diskutiert werden, aber auch Gefahren durch unangemessene Formen der Kommunikation durch die Jugendlichen selbst, z. B. Cybermobbing (vgl. die systematische Problemauflistung bei Hans-Bredow-Institut für Medienforschung 2014), gezielte Fehlinformationen, die Verletzung der Persönlichkeitsrechte oder des Datenschutzes. Vermehrt bergen die in den virtuellen Welten erfolgten Selbstäusserungen und Selbstdarstellungen der Heranwachsenden die Gefahr, dass sie durch Dritte, durch Anbieter oder Netzbetreiber beobachtet, für eigene oder fremde Zwecke ausgewertet oder sogar gegen die Jugendlichen selbst verwendet werden (vgl. dazu als drastisches Beispiel das Dossier «Lauras Entblößung» in der Zeit Nr. 
26 vom 18. Juni 2014: 13-15). Schliesslich besteht die Gefahr, dass sich viele Kinder und Jugendliche in ihrem Medienhandeln durch die algorithmische Steuerung der Kommunikationsprozesse in den Plattformen, Apps und Computerspielen manipulieren lassen (z. B. Radikalisierung im Netz) und für ideologische oder ökonomische Interessen ausgebeutet werden. Dabei besteht die Gefahr, dass sich die Heranwachsenden in den faszinierenden medialen Spielwelten verlieren, mit Begeisterung die Anforderungen eines Spiels erfüllen, dabei aber die Ansprüche ihrer Alltagswirklichkeit und die Arbeit an ihren Entwicklungsaufgaben vernachlässigen (Spanhel 2006, $144 \mathrm{ff}$.).

Diesen neuen Gefährdungen kann durch gesetzliche Regelungen des Kinder-und Jugendmedienschutzes allein nicht Rechnung getragen werden. Sie müssen durch pädagogisch gestaltete lebensweltliche Kontexte in Familie und Freizeit und eine systematische medienpädagogische Unterstützung in den Bildungseinrichtungen ergänzt werden. Gezielte medienpädagogische Massnahmen sind unverzichtbar, damit die Heranwachsenden Reflexionsfähigkeit und Medienbildung erwerben sowie eine Präferenzordnung mit klaren Sinnkriterien für die Selektion von Medienangeboten und Medienhandlungsmustern aufbauen und sich selbst ein Stück weit vor möglichen Gefährdungen schützen können. Eine kontextuelle Steuerung des Medienhandelns der Heranwachsenden bietet nach meiner Ansicht die beste Möglichkeit für eine wirkungsvolle Kooperation von Medienschutz und Medienpädagogik.

\section{Zusammenwirken von Medienschutz und Medienpädagogik im Rahmen einer kontextuellen Steuerung des Medienhandelns der Heranwachsenden}

\subsection{Die Doppelfunktion der Selbstregulationsprozesse}

Die Systemtheorie geht von der Annahme aus, dass zwischen Systemen alle Prozesse in Form von Rückkopplungsschleifen ablaufen. Das bedeutet, dass z. B. die Auswirkungen medienpädagogischer Massnahmen der 
Eltern oder familiärer Regelungen zur Mediennutzung auf das Medienhandeln der Kinder häufig durch deren eigensinnige Medienhandlungsmuster konterkariert werden und diese auf den Medienumgang in der Familie zurückwirken. Grundsätzlich gilt daher: Heranwachsende bestimmen durch ihr selbstgesteuertes Medienhandeln die Kontexte mit, in denen sie heranwachsen und erzogen werden. In diesem Sinne sind Heranwachsende immer auch Subjekte ihrer eigenen (Medien-)Erziehung. Und die digitalen Medien geben ihnen bisher nie gekannte Möglichkeiten zur eigenwilligen Gestaltung ihrer lebensweltlichen Kontexte und zur Konstruktion eigener virtueller Realitäten.

Wenn das Medienhandeln der Heranwachsenden durch pädagogische Gestaltung ihrer lebensweltlichen Kontexte, d. h., durch Ausrichtung auf pädagogisch begründete, auf Entwicklungsförderung zielende Sinnorientierungsmöglichkeiten gesteuert werden soll, ergibt sich folgendes Problem: Diese Steuerung ist mühsam und ungenau, weil sie stets die Selbststeuerung der Heranwachsenden und deren Rückwirkungen auf die Steuerungsinstanzen (Familie, Kita, Schule) berücksichtigen muss. Zudem befinden sich auch diese Erziehungsinstitutionen selbst in einem Entwicklungsprozess, an den die Entwicklung der Heranwachsenden gekoppelt ist (Ko-Ontogenese). Die Kopplung erzwingt eine ständige Abstimmung dieser Entwicklungsprozesse aufeinander und stellt eine eigene pädagogische Aufgabe dar, die in jeder konkreten Handlungssituation neu bewältigt werden muss. Diese Abstimmung kann gelingen, wenn den Heranwachsenden die Möglichkeit gegeben wird, ihr Medienhandeln zur Verwirklichung ihrer eigenen Bedürfnisse, Ziele und Wünsche selbst so zu regulieren, dass sie gleichzeitig auch den Erwartungen des sozialen Systems gerecht werden, in dem sie gerade handeln.

Erziehungsinstitutionen müssen daher bei ihren medienpädagogischen Massnahmen die Bedürfnisse, Anliegen und Wünsche der Kinder und der Jugendlichen ernst nehmen und sich einerseits auf ihre Selbststeuerungsfähigkeit einstellen und andererseits dabei stets die Weiterentwicklung dieser Fähigkeit unterstützen. Diese unterschiedlichen pädagogischen Sinnorientierungen müssen aufeinander abgestimmt und in ein Fliessgleichgewicht gebracht werden, damit die Heranwachsenden in ihrem Medienhandeln sowohl ihren eigenen Entwicklungsbedürfnissen 
und Interessen als auch den Erfordernissen und Erwartungen des sozialen Systems gerecht werden können. (Mit Gleichgewicht sind hier im Sinne von Piaget (1980, 271 ff.) aktive Kompensationen zum Ausgleich von Störungen gemeint.) Dies erscheint nur dann möglich, wenn das selbstgesteuerte Medienhandeln im Rahmen der durch die sozialen Kontexte eingegrenzten Sinnorientierungsmöglichkeiten erfolgt. Die pädagogisch Verantwortlichen müssten sich also darum bemühen, die alltagsweltlichen Kontexte so zu gestalten, dass die Kinder und Jugendlichen innerhalb dieser Rahmen ihr Medienhandeln an Sinnorientierungen ausrichten, die ihre Entwicklung fördern. Nur auf diese Weise kann auch im Gesamtzusammenhang der Gesellschaft mit ihren rasanten Medienentwicklungen und der fortschreitenden Mediatisierung aller Lebensbereiche sowohl der Bildungsprozess der Heranwachsenden, ihre gedeihliche Entwicklung und ihre soziale Integration als auch die Weiterentwicklung und Stabilisierung der sozialen Systeme der Gesellschaft und ihrer Medienkultur gesichert werden (Spanhel 2014, 140 f.).

\subsection{Notwendigkeit eines Zusammenwirkens von Kinder- und Jugendmedienschutz und Medienpädagogik}

Die Notwendigkeit für ein Zusammenwirken von Medienschutz und $\mathrm{Me}$ dienpädagogik ergibt sich nun aus der schwierigen Aufgabe, diese Doppelfunktion der Selbstregulationsprozesse im Medienhandeln der Heranwachsenden zur Geltung zu bringen. Damit sind Anforderungen verbunden, die nur im Zusammenspiel von Medienschutz und Medienpädagogik erfüllt werden können:

Die gesetzlichen Vorgaben des Kinder- und Jugendmedienschutzes müssen in den alltagsweltlichen Kontexten konsequent befolgt und den Kindern und Jugendlichen in ihrer Sinnhaftigkeit und Bedeutung verständlich gemacht werden. Der Medienschutz wirkt dann als ein eigenes symbolisches Sinnsystem, als ein übergeordneter, gesetzlich verankerter und verbindlicher Kontext. Er begrenzt das Medienhandeln dadurch, dass in den Lebensräumen der Heranwachsenden bestimmte Sinnorientierungen und Handlungsmöglichkeiten von Gesetzes wegen ausgeschlossen werden, die den zentralen Werten des Grundgesetzes und den 
Grundrechten der Heranwachsenden widersprechen, ihre Würde verletzen oder ihre Entwicklung oder ihre soziale Teilhabe gefährden. Dieser Rahmen ist für alle lebensweltlichen Kontexte, für alle pädagogischen Institutionen und Handlungsrahmen verbindlich. Daher sind die gesetzlichen Bestimmungen des Medienschutzes auch sanktionsbewehrt, mit allen Problemen, die die entsprechenden Institutionen des Medienschutzes und ihre juristischen und administrativen Verfahren auf Bundes- und Länderebene mit sich bringen. (Zur Problematik der Begrenzung und Öffnung von Mediensystemen vgl. Leschke 2012). Die Medienpädagogik müsste auf der Grundlage medienpädagogischer Forschung und Theoriebildung ihren Beitrag für die Begründung, Legitimierung und Ausdifferenzierung dieser gesetzlichen Bestimmungen leisten. Sie müsste aber mit noch viel grösserem Nachdruck dafür kämpfen, dass die gesetzlichen Bestimmungen des Medienschutzes sowie die zuständigen Institutionen und die Verfahrensregeln zur Durchsetzung dieser Bestimmungen vereinfacht und klarer formuliert werden, damit sie nicht nur für spezialisierte Juristen, sondern auch für die pädagogisch Verantwortlichen und für die Heranwachsenden verständlich und nachvollziehbar sind.

Durch die gesetzliche Begrenzung von Sinnorientierungsmöglichkeiten entstehen geschützte Räume, in denen für die Heranwachsenden erst sinnorientiertes Handeln mit der Chance einer Verständigung möglich wird. Aus dieser Perspektive darf der Medienschutz nicht als die Errichtung eines lebensfremden pädagogischen Schonraums missverstanden werden. Vielmehr sichert er in einem positiven Sinn die grundlegenden, gemeinsamen Wertorientierungen in unserer Gesellschaft, d. h. die Ausrichtung der Entwicklung der Heranwachsenden am Menschenbild des Grundgesetzes (Baum 2008, 60:117ff; 182 ff.). Solche «befriedeten Räume» sind ausserordentlich wichtig, weil durch die zunehmende Entgrenzung aller Lebensbereiche der Heranwachsenden in der Folge der Mediatisierung mit ihrer Vielfalt an scheinbar gleich-gültigen Angeboten an Wertorientierungen, Lebensstilen und Weltauffassungen klare Orientierungen für sinnvolles Medienhandeln verloren zu gehen drohen (Koziol 2000).

Für die Medienpädagogik erwächst daraus die Aufgabe, den pädagogisch Verantwortlichen sowie den Heranwachsenden diese Medienschutzbestimmungen in ihrer gesellschaftlichen und individuellen Bedeutung 
bekannt und so verständlich zu machen, dass sie von ihnen auch akzeptiert werden können. Kinder und Jugendliche müssen sich an den Begrenzungen reiben, sich mit diesen normativen Vorgaben auseinandersetzen, um zu eigenen Sinnorientierungen zu gelangen und die Fähigkeit zu eigenverantwortlichem Handeln zu erwerben. Aus pädagogischer Sicht müssten die gesetzlichen Regelungen und Massnahmen des Medienschutzes selbst dann beibehalten werden, wenn sie nicht alle Gefährdungsmomente wirkungsvoll ausschalten können und auch, wenn die Heranwachsenden sich immer wieder darüber hinwegsetzen. Sie wirken als ein normatives Regulativ im Sinne einer Steuerungsgrösse, um mögliche Gefährdungen für eine gedeihliche Entwicklung der jungen Menschen bei pädagogisch Verantwortlichen und bei Heranwachsenden im Bewusstsein zu halten. An diesen Grenzen müssen sich daher auch Eltern und pädagogische Fachkräfte in ihren medienpädagogischen Massnahmen orientieren.

Der Medienschutz konstituiert auf der einen Seite geschützte Räume, die Sicherheit, Ordnung und Überblick bieten, auf der anderen Seite gewährt er im Inneren immer noch grosse Freiräume für das Medienhandeln der Heranwachsenden, weil die gesetzlichen Regelungen aus den grundsätzlich möglichen Sinnorientierungen nur wenige ausgrenzen. Deshalb ist es unabdingbar, dass die Medienerziehung in den alltagsweltlichen Kontexten (Familie, Kita, Schule) ergänzend zu den gesetzlichen Grenzen des Medienschutzes weitere, pädagogisch begründete Begrenzungen möglicher Sinnorientierungen festlegt, z. B. in Form von Regeln oder Absprachen (Hasebrink 2014). Sie müssen sich aber nun viel genauer an der konkreten Lebenssituation, am Entwicklungsstand, der Handlungsfähigkeit und Selbstschutzfähigkeit der Heranwachsenden orientieren. Wichtig sind dabei überzeugende und verständliche Begründungen solcher Begrenzungen gegenüber den Kindern und Jugendlichen und - soweit möglich - das gemeinsame Aushandeln von Grenzsetzungen, die entsprechend ihrem Entwicklungsprozess immer mehr ausgeweitet werden müssen.

Für die Medienpädagogik stellt sich damit als weitere fundamentale Aufgabe, diese entwicklungsnotwendigen Handlungsspielräume zu sichern, in denen Kinder und Jugendliche selbstbestimmt, lustvoll und spielerisch lernen können. Diese Spielräume dürfen ihnen nicht durch rigorose, unbegründete oder willkürliche Verbote und Kontrollen genommen werden, 
denn nur wenn ihnen Autonomie gewährt wird, können die Heranwachsenden eigene Wertorientierungen und die Fähigkeit zu eigenverantwortlichem Medienhandeln aufbauen. In der gemeinsamen Gestaltung dieser Freiräume für Medienhandeln liegen die weiteren Aufgaben und Handlungsfelder der Medienpädagogik, wie sie bisher im Sinne einer umfassenden Medienkompetenzförderung verstanden wurden (Tulodziecki, Herzig, und Grafe 2010), um die Kinder und Jugendlichen zu einem kritischreflexiven Medienhandeln zu befähigen und ihr Medialitätsbewusstsein (Pietraß 2014) zu fördern.

Die Notwendigkeit für ein abgestimmtes Zusammenwirken von Kinder- und Jugendmedienschutz und Medienpädagogik ergibt sich also aus dem Zusammenhang von Eingrenzung der Sinnorientierungsmöglichkeiten, Autonomiegewährung für selbstgesteuertes Medienhandeln und (medien-)pädagogischen Hilfen für den Aufbau von Medienkompetenz.

Dieser Zusammenhang bildet das Fundament für ein gedeihliches Aufwachsen, das sich weder allein durch gesetzliche Regelungen oder pädagogische Programme, noch allein durch das Vertrauen auf die Medienkompetenz, Selbststeuerungs- und Selbstschutzfähigkeit der Heranwachsenden sichern lässt. Er lässt sich nach meiner Überzeugung am ehesten durch eine Ausrichtung der Lebensräume der Kinder und Jugendlichen (Familie, Kita, Schule) an entwicklungsförderlichen Sinnorientierungen in Form einer «kontextuellen Steuerung des Medienhandelns» herstellen. Auf gesamtgesellschaftlicher Ebene wird ein abgestimmtes Zusammenwirken von Begrenzung, Autonomiegewährung und pädagogischer Unterstützung in einer digitalisierten Lebenswelt insbesondere auch durch die für Deutschland als verbindlich erklärte UN-Kinderrechtskonvention legitimiert, die sehr differenziert die Schutz-, Teilhabe- und Förderrechte der Kinder als unverzichtbare Bedingung für ihr gedeihliches Aufwachsen beschreibt (Stapf 2018). 


\subsection{Medienpädagogisches Handeln als kontextuelle Steuerung des Medienhandelns}

Die Bedingungen der Möglichkeit für eine kontextuelle Steuerung des Medienhandelns der Heranwachsenden liegen im Zusammenwirken von gesetzlichen Regelungen mit medienpädagogischen Massnahmen. Das Konzept der «regulierten Selbstregulierung» als Grundlage für den Medienschutz in Deutschland, das traditionelle Steuerungsmittel in Form von Verboten und Strafen mit der Eigenverantwortung der Betroffenen verbindet (Bosch 2007, 383), bietet dafür eine Basis. Mit kontextueller Steuerung ist hier die Gestaltung der sinnstiftenden Kontexte gemeint, aus denen die Heranwachsenden die Sinnorientierungen für ihr Medienhandeln auswählen (Zur Problematik des Begriff der Steuerung bei komplexen Systemen vgl. Willke 1991, 146; 2005, 111ff.; 286; 296).

Kontextuelle Steuerung des Medienhandelns wird durch ein abgestimmtes Zusammenwirken von Grenzsetzungen, Autonomiegewährung und medienpädagogischen Massnahmen möglich (Retzke 2007).

Leider sind in unserer heutigen Gesellschaft Konzepte, die auf Grenzsetzung, auf pädagogische «Eingriffe» oder Erziehung abzielen, oft sehr stark mit negativen Einschätzungen und Gefühlen verbunden. Von Erziehung ist heute kaum noch die Rede, weil damit fälschlicherweise häufig Vorstellungen von Einschränkung oder gar Unterdrückung der Freiheit, von Aussenlenkung und Fremdsteuerung der Heranwachsenden assoziiert werden. Damit hat das Konzept einer kontextuellen Steuerung nichts zu tun. Es handelt sich vielmehr um eine Form der indirekten Steuerung, durch die mögliche Sinnorientierungen ausgeschlossen werden sollen, die die Entwicklung der Heranwachsenden gefährden oder beeinträchtigen könnten. Entscheidend ist, dass der Sinn dieser Grenzsetzungen in der notwendigen Begrenzung von Sinn liegt, um der Entwicklungstatsache der Heranwachsenden Rechnung zu tragen: Nur in eingegrenzten Freiräumen können Kinder und Jugendliche die Wahrnehmungs-, Gefühls-, Bewertungs-, Denk- und Handlungsmuster aufbauen und in sich vergrössernden Freiräumen kontinuierlich weiterentwickeln, die die Voraussetzung für ein sozial verträgliches, selbstbestimmtes und verantwortungsbewusstes Medienhandeln bilden. Entscheidend ist die Einsicht: 
Selbstregulation des Medienhandelns und seine kontextuelle Steuerung stellen keinen Widerspruch dar, sondern bieten den einzig möglichen Rahmen für medienpädagogisches Handeln:

- Kontextuelle Steuerung richtet sich nicht direkt auf das Medienhandeln der Kinder und Jugendlichen, sondern auf die pädagogische, d. h., entwicklungsförderliche Gestaltung relevanter Umgebungen.

- Kontextuelle Steuerung bedeutet eine Ausrichtung des Medienhandelns der Heranwachsenden durch Eingrenzung der Sinnorientierungsangebote, aber keine direkte Bestimmung ihres Handelns.

- Kontextuelle Steuerung nimmt die doppelte Kontingenz aller Kommunikationsprozesse in sozialen Systemen in Kauf, die auf der pädagogischen Seite mit Ungewissheit und Risiko verbunden ist, den $\mathrm{He}$ ranwachsenden aber die entwicklungsnotwendigen Freiräume für die Selbststeuerung ihres Medienhandelns belässt.

Medienpädagogisches Handeln als kontextuelle Steuerung des $\mathrm{Me}$ dienhandelns beruht auf den drei sich wechselseitig ergänzenden Komponenten Begrenzung der medialen Sinnorientierungsangebote, Autonomiegewährung und medienpädagogische Massnahmen. Damit realisiert sich in einer kontextuellen Steuerung des Medienhandelns der Heranwachsenden das als notwendig erkannte, abgestimmte Zusammenwirken von $\mathrm{Me}$ dienschutz und Medienpädagogik, denn in der Komponente der Grenzsetzung ist die Grundidee des Medienschutzes impliziert. Die Notwendigkeit der Grenzsetzungen und damit verbundene Aufgaben der Medienerziehung wurden bereits erläutert. Aber Grenzsetzungen und Autonomiegewährung müssen immer wieder neu ins Verhältnis gesetzt werden: Wie können Prozesse der Radikalisierung im Netz verhindert oder der Zugang zu Gewalt verherrlichenden Computerspielen unterbunden werden, ohne die Heranwachsenden von bereichernden Erfahrungen abzuschneiden? Wie kann eine Begrenzung der Handynutzung den Schülern einer Klassenstufe überzeugend begründet werden, um ständige Ablenkungen beim Lernen und Störungen des Unterrichts zu vermeiden? Wie können gemeinsam Regelungen gefunden werden, um die Möglichkeiten des Smartphones zur Verbesserung der Lehr- und Lernprozesse zu nutzen? Im Alltag zeigt sich die Verunsicherung vieler Eltern, Lehrer und Erzieher hinsichtlich der 
Frage, wo Grenzen gesetzt und wieviel Freiheiten beim Medienumgang gewährt werden sollten: Die einen scheuen sich oder fühlen sich überfordert, überhaupt noch Grenzen zu setzen und lassen die Kinder bei ihrer Mediennutzung völlig frei gewähren; die anderen wollen zum Wohle ihres Kindes alles regeln und das Medienhandeln der Kinder komplett kontrollieren, was prinzipiell nicht möglich ist.

Eine Möglichkeit sind klare Grenzsetzungen und die Gewährung von Handlungsfreiräumen entsprechend dem Alter der Heranwachsenden sowie ihre Sicherung (z. B. durch Absprachen). In diesem Zusammenhang muss bedacht werden, dass gerade die digitalen Medien den Heranwachsenden trotz aller gut gemeinten pädagogischen Kontrollen im Alltag genügend Gelegenheiten bieten, sich Freiräume und ihre eigenen medialen Handlungsrahmen zu schaffen - unbemerkt oder gegen getroffene Absprachen. In beiden Fällen bestimmen die Heranwachsenden selbst das Verhältnis zwischen den zwei Ebenen der virtuellen und der realen Wirklichkeit und die Art und Weise, die sich gegenseitig in ihren Bedeutungen definieren: Die Erfahrungen ihrer Alltagssituation verleihen der virtuellen Realität spezifische Bedeutungen und umgekehrt wirken die eindrucksvollen Medienerfahrungen darauf ein, wie Kinder oder Jugendliche ihre Alltagssituation erleben. Die Heranwachsenden wollen schon sehr früh und soweit irgend möglich dieses Verhältnis selbst kontrollieren. Das ermöglicht ihnen spezielle Formen von Erfahrungen, die sich von den pädagogisch gesteuerten und kontrollierten Lern- und Bildungsprozessen in Kita, Familie, Schule oft stark unterscheiden. Diese Erfahrungen können die selbständige Bewältigung ihrer Entwicklungsaufgaben stabilisieren, die Selbstachtung der Heranwachsenden fördern und durch vielfältige Identifikationsangebote den Prozess der Identitätsbildung unterstützen. Aber diese Prozesse sind während des gesamten Entwicklungsverlaufs mehr oder weniger prekär, besonders in den schwierigen Übergangsphasen vom Kind zum Jugendlichen und vom Jugendlichen zum Erwachsenen, ebenso auch in problematischen persönlichen Lebenssituationen, z. B. bei familiären Konflikten, Schulschwierigkeiten, Krankheiten, Ausgrenzung aus der Peergroup. Fast täglich können wir Presseberichte über schwer wiegende Entwicklungsstörungen und Fehlentwicklungen bei jungen Menschen lesen, die aus ihren Medienwelten nicht mehr herausgefunden haben. 
In solchen Situationen, aber auch wenn Grenzen missachtet oder mutwillig überschritten werden, wenn Kinder oder Jugendliche sichtbar Schwierigkeiten mit bestimmten Medien haben, von sich aus Hilfe anfordern oder die gewährten Freiräume für einen Medienumgang nutzen, der offensichtlich ihrer Entwicklung schadet, dann ist pädagogisches Handeln gefordert. Daraus resultiert eine weitere fundamentale Aufgabe medienpädagogischen Handelns: eine aufmerksame Beobachtung und zurückhaltende Begleitung des Medienhandelns der Kinder und Jugendlichen in diesen Autonomieräumen. Zunächst ist es wichtig, ihr Medienhandeln vorbehaltlos und nicht wertend zu beobachten und bei Unsicherheiten, Konflikten oder Grenzübertretungen Hilfen anzubieten. Eine kontinuierliche Begleitung verlangt dann von den pädagogisch Verantwortlichen (Eltern, Erziehende, Lehrpersonen, Sozialpädagoginnen und -pädagogen, oder Medienpädagoginnen und -pädagogen), dass sie ihre Beobachtungen den Heranwachsenden zurückmelden, mit ihnen darüber sprechen, um ihre Sichtweisen zu erfahren und gemeinsam mit ihnen nach Handlungsalternativen suchen. Ihre pädagogischen Bemühungen sollten sich dabei von Anfang an auf die Unterstützung der Selbstregulationsprozesse der Heranwachsenden richten (Spanhel 2014, 135 ff.). Bei medienpädagogischer Massnahmen und Projekten kommt es darauf an, positive Entwicklungsanreize und überzeugende Handlungs- und Wertorientierungen zu setzen. Nur durch die Auseinandersetzung mit klaren Normen und Anforderungen im Zusammenhang mit beispielhaften Medienangeboten können die Kinder und Jugendlichen die Muster ihrer inneren Strukturen ausdifferenzieren, stabilisieren und alternative Medienhandlungsmuster ausbilden. Dabei können sie ihre Wahrnehmungsmuster und ihr ästhetisches Empfinden, ihre Sensibilität für Medienwirkungen bei sich und anderen, ihr Wissen über die Welt verbessern und ein breites Interessenspektrum ausbilden. Je differenzierter und flexibler diese Strukturen des psychischen Systems und je stabiler eine an den Grundwerten orientierte Präferenzordnung als zentrale Steuerungsgrösse für die Selbstregulationsprozesse, desto besser können sie Anforderungen ihrer komplexen Medienumwelt selbständig bewältigen und positive Anregungen für ihren Entwicklungsprozess gewinnen. 
Kontextuelle Steuerung des Medienhandelns der Heranwachsenden im Sinne einer Abstimmung der drei Komponenten der Grenzsetzung, Autonomiegewährung und pädagogischer Unterstützung muss sich insbesondere auf die mediale Gestaltung der einzelnen alltagweltlichen Kontexte als übergreifende pädagogische Handlungsrahmen richten. Die Heranwachsenden werden auf diese Weise unterstützt und lernen mehr und mehr selbst, wie sie ihre medialen Handlungsrahmen zeitlich und räumlich in die Kontexte ihrer Alltagswelt in Familie, Kita, Schule und Jugendarbeit integrieren sollen. Wie soll diese Integration unter Berücksichtigung ihrer Medieninteressen in einer für alle Beteiligten befriedigenden Weise geregelt werden? Unter Anerkennung der Selbstregulation ihres Medienhandelns liegen besonders gute Möglichkeiten dafür in der gemeinsamen medialen Gestaltung dieser Kontexte. Die Bedeutung, die den digitalen Medien für das Zusammenleben in Familie, Kindertagesstätte, Schulklasse zugesprochen wird, sollte gemeinsam mit den Kindern und Jugendlichen entsprechend ihrem Verständnis geklärt, begründet und mit zunehmendem Alter mit ihnen ausgehandelt werden. Dieses gemeinsame Bemühen um übereinstimmende Bewertungen der Medien, Medienangebote und Medienhandlungsmuster und um gemeinsame Sinnorientierungen für den Medienumgang bildet eine gute Basis für ein gedeihliches Aufwachsen in digitalisierten Lebenswelten (Spanhel 2018). 


\section{Praxiskonzept für eine Kontextsteuerung des Medienhandelns im Zusammenwirken von Medienschutz und Medienpädagogik}

\subsection{Prinzipien einer kontextuellen Steuerung des Medienhandelns}

Kontextuelle Steuerung des Medienhandelns durch mediale Gestaltung relevanter Kontexte sollte den Heranwachsenden die Möglichkeit geben und sie mit zunehmendem Alter befähigen, die leitenden Sinnorientierungen ihres Medienhandelns zu erkennen, sich bewusst zu machen und zu reflektieren. Auf dieser Basis können sie lernen, in ihrem Medienhandeln eine Abstimmung zwischen ihren eigenen Bedürfnissen, Interessen, Wünschen und Zielen und den im jeweiligen Kontext geltenden sozialen Normen, Regeln und Routinen vorzunehmen. Rationales, eigenverantwortliches Medienhandeln heisst, diesen Ausgleich bewusst anzustreben. Pädagogische Begleitung und Unterstützung heisst, gemeinsam mit den Heranwachsenden einen für ihre Entwicklung förderlichen Ausgleich zwischen divergierenden Sinnorientierungen zu suchen und zu finden. Kontextuelle Steuerung

- zielt auf das Angebot, die Begründung und die Vermittlung von entwicklungsförderlichen Sinnorientierungen, die das selbstgesteuerte Medienhandeln der Heranwachsenden unterstützen;

- erfordert auf allen Kontextebenen ein immer neues Austarieren zwischen Rahmensetzungen, Autonomiegewährung und medienpädagogischen Angeboten und Orientierungshilfen;

- orientiert sich an der Selbstregulationsfähigkeit der Kinder und Jugendlichen und fördert die Entwicklung ihrer inneren Strukturen als Steuerungsgrössen;

- fördert ihre Fähigkeit zur Medienkritik (Spanhel 2018), zu rationalem Medienhandeln und zur Abschätzung der Handlungsfolgen durch das gemeinsame Aushandeln von Grenzsetzungen und die gemeinsame Gestaltung von Handlungsrahmen; 
- gewährt eine schrittweise Ausdehnung der Autonomie als Voraussetzung für die Erlangung intellektueller und moralischer Autonomie der Heranwachsenden.

\subsection{Ansatzpunkte und Steuerungsgrössen für eine kontextuelle Steuerung des Medienhandelns}

Aus Abbildung 1 ist ersichtlich, dass es zwei Ansatzpunkte gibt, um das Medienhandeln an pädagogisch begründeten Sinnorientierungen auszurichten: Das sind die beiden Selektionen, zum einen die Auswahl aus den Sinnorientierungsangeboten (Selektion 1), um dem Medienhandeln eine subjektive Bedeutung zu geben und zum anderen die Auswahl aus den Medienhandlungsmöglichkeiten (Selektion 2), um diesen subjektiven Sinn verwirklichen zu können. Weil die Medienangebote für die Heranwachsenden erst im Kontext ihre je spezifisch subjektive Bedeutung erlangen, kann ihr Medienhandeln als Ergebnis der beiden Selektionsprozesse durch die mediale Gestaltung der Kontexte - wenn auch nur indirekt - gesteuert werden.

Eine indirekte Steuerung der ersten Auswahlprozesse (Selektion 1) lässt sich durch eine Begrenzung der Sinnorientierungsangebote der sozialen Kontexte erreichen. Wichtigste Steuerungsgrösse dafür ist die medienpädagogische Kompetenz bei den pädagogisch Verantwortlichen in den Erziehungs- und Bildungseinrichtungen, also bei Eltern, Erziehende, Lehrpersonenn (Hasebrink, Schröde, und Schumacher 2012; Wagner u. a. 2013). Dazu gehören ein Wissen um die Chancen und Gefahren des Medienhandelns im Entwicklungsprozess sowie die Bereitschaft zur Übernahme der Verantwortung für eine entwicklungsförderliche Gestaltung medialer Handlungsrahmen in den unterschiedlichen Lebenssituationen der Kinder und Jugendlichen. Das setzt zwingend ein medienpädagogisches Grundwissen für Eltern und eine medienpädagogische Aus- und Fortbildung für alle pädagogischen Fachkräfte voraus. Sie müssen besonders sensibel darauf achten, welche Sinnorientierungen für die Heranwachsenden in ihrer jeweiligen Entwicklungssituation besondere Bedeutung haben. Diesen Bedeutungszuschreibungen müssen sie bei allen Grenzsetzungen Raum geben. Empirische Untersuchungen zeigen, dass sich die Heranwachsenden 
bei ihrer Auswahl aus den bereits eingegrenzten Sinnorientierungsangeboten von drei Meta-Kontexten leiten lassen, in denen sie sich in ihrer aktuellen Lebenssituation am stärksten unter Druck fühlen: Die Entwicklungsaufgaben, die sich in bestimmten Entwicklungsabschnitten unausweichlich stellen und die sie selbständig bewältigen müssen; die Mitgliedschaft in einer Peergroup, in der sie sich auf ihrem Weg ins Erwachsenenleben Orientierung und Anerkennung holen, weil sie sich mehr und mehr von der elterlichen Autorität ablösen müssen und schliesslich die Teilhabe an den faszinierenden Medienangeboten, mit deren Hilfe sie ihre eigene Alltagswelt nach ihren subjektiven Bedürfnissen, Vorstellungen und Wünschen immer wieder neu gestalten können (Spanhel 2006, 156 ff.). Diese Sinn stiftenden Meta-Kontexte müssen bei einer pädagogischen Gestaltung der Alltagskontexte berücksichtigt werden.

Bei der Regulierung der zweiten Auswahlprozesse (Selektion 2) im psychischen System kommt es darauf an, über welche Medienhandlungsmuster die Heranwachsenden überhaupt verfügen und welche sie in der konkreten Situation einsetzen können, um die im ersten Selektionsprozess ausgewählte Sinnorientierung zu verwirklichen. Die Chancen für ein entwicklungsförderliches Medienhandeln sind umso grösser, je mehr alternative Medienhandlungsmuster sich die Heranwachsenden angeeignet haben und je besser sie in der Lage sind, die inneren Regulationsprozesse rational zu kontrollieren. Die Entscheidung für ein bestimmtes Medienhandeln ist Ergebnis der Regulationen zwischen den basalen Trieben (Bewegungsdrang, Neugier, Aggressivität), der Befriedigung grundlegender Bedürfnisse (Sicherheit, Geborgenheit, Zuwendung, Anerkennung), der Stimmungen und Gefühle (Langeweile, Freude, Ärger, Spannung) sowie der kognitiven Fähigkeiten, Interessen und Ziele. Und diese psychischen Faktoren sind zusätzlich noch mit körperlichen Befindlichkeiten (Anspannung, Müdigkeit, Kraft, Geschicklichkeit), also mit dem biologischen System des Menschen gekoppelt.

Diese inneren Regelungsprozesse beruhen immer auf Beurteilungen, Regeln, Routinen und Mustern, die von den Kindern und Jugendlichen erst im Verlaufe ihres Entwicklungsprozesses selbst konstruiert, ausdifferenziert und immer wieder umstrukturiert werden. Dahinter steht eine zentrale Steuerungsgrösse, die sich an der Erhaltung und Entwicklung der 
Identität orientiert, eine eigene Präferenzordnung, ein System von Wertorientierungen, das im Verlaufe der Identitätsentwicklung aufgebaut, immer wieder umgebaut und neu stabilisiert werden muss (Spanhel 2013a).

Die inneren Regulationsprozesse sind weder beobachtbar, noch pädagogisch kontrollierbar. Erst wenn ihre Ergebnisse als konkretes Medienhandeln sichtbar werden, können sie durch die weitere Gestaltung der alltagsweltlichen Kontexte in Richtung auf ein entwicklungsförderliches $\mathrm{Me}$ dienhandeln gelenkt werden. Im Alltag kommt es dabei häufig zu Diskrepanzen zwischen widersprüchlichen Bewertungen eines Medienangebots oder eines Medienhandlungsmusters zwischen den Medienanbietern, den Eltern oder Gleichaltrigen auf der einen und den Heranwachsenden auf der anderen Seite. In der Bewältigung dieser Differenzen liegen die Chancen für eine Ausdifferenzierung und Neustabilisierung der Präferenzordnung und für eine systematische Integration von Wertorientierungen, die sich auf das Medienhandeln beziehen. Eine kontextuelle Steuerung bietet die Chance, in einem offenen Kommunikationsprozess im Rahmen einer vertrauensvollen Beziehung diese Differenzen zu erkennen, zu bearbeiten und zu gemeinsamen, entwicklungsförderlichen Wertorientierungen zu gelangen. Medienpädagogische Massnahmen und Programme müssten sich daher primär um die Befähigung zur Kritik der Medienangebote, zur kritischen Reflexion des eigenen Medienhandelns und zur Beurteilung möglicher Handlungsfolgen bemühen (Spanhel 2018).

\subsection{Praktische Möglichkeiten einer Kontextsteuerung des Medienhandelns}

Im Folgenden kann nur sehr allgemein und beispielhaft aufgezeigt werden, welche Ansatzpunkte, Massnahmen und methodische Vorgehensweisen für eine kontextuelle Steuerung des Medienhandelns in der Praxis geeignet erscheinen. Angesichts der beschriebenen Komplexität und Einmaligkeit jeder Situation können Entscheidungen für die konkrete Vorgehensweise nur innerhalb der Situation getroffen werden. Dabei geht es vor allem um den Aufweis von Handlungsalternativen. 
Massnahmen zur Rahmensetzung für Medienhandeln:

- (Rahmensetzung meint im Folgenden immer die Begrenzung von Sinnorientierungsmöglichkeiten)

- Kontextebene Gesellschaft: Begrenzung durch die Regelungen und Massnahmen des gesetzlichen Kinder- und Jugendmedienschutzes

- Kontextübergreifend: Begrenzung individueller Handlungsrahmen durch technische Vorrichtungen mit Bezug auf die gesetzlichen Regelungen (Jugendschutzprogramme)

- Kontextebene Familie und Freizeit: Begrenzung durch bewusste mediale Gestaltung des alltäglichen familiären Zusammenlebens und durch reflektierten Medienumgang

- Kontextebene pädagogische Institutionen (Kita, Schule, Horte, Heime): Begrenzung durch Programme und Massnahmen zur Medienkompetenzförderung und Medienbildung

- Situative Kontexte: Individuelle Begrenzung medialer Handlungsrahmen in konkreten Lebenssituationen durch Vorgabe oder Aushandeln von Regeln, Geboten oder Verboten entsprechend dem Entwicklungsstand und der Lebenssituation der einzelnen Kinder oder Jugendlichen.

Massnahmen der Autonomiegewährung als Möglichkeitsbedingung für eigenverantwortliches Medienhandeln:

- Ermöglichung, Bereitstellung und Sicherung von entwicklungsnotwendigen Handlungsspielräumen

- für Erfahrungen mit selbst konstruierten medialen Handlungsrahmen zur Unterhaltung und Informationsgewinnung, aber auch zum Spiel

- für Erfahrungen mit unterschiedlichen Kommunikationsformen bei der Teilnahme an sozialen Medien und Netzwerken und zur medialen Selbstdarstellung

- zur Erprobung und Festigung erworbener Medienkompetenzen im Rahmen von Medienprojekten und bei der Produktion eigener Medien

- zur Befriedigung elementarer Grundbedürfnisse, subjektiver Wünsche und zur Entwicklung breiter Interessen als Voraussetzung für rationales, eigenverantwortliches Medienhandeln

- zur Stärkung der subjektiven Fähigkeiten für die autonome und verantwortliche Gestaltung medialer Handlungsrahmen. 
Massnahmen pädagogischer Begleitung und Unterstützung bei der Entwicklung der Fähigkeit zur reflexiven Steuerung des Medienhandelns:

- Anregungen durch Medienangebote, die sich an den jeweiligen Bedürfnissen, Interessen, Wünschen und Zielen der Heranwachsenden orientieren und ihnen vielfältige Medienhandlungsmuster eröffnen

- Programme und Massnahmen zur Förderung der Medienkompetenz, wie sie seit Jahren in medienpädagogischen Institutionen und Bildungseinrichtungen erarbeitet und erprobt wurden

- Erprobung alternativer Handlungsrahmen durch praktische Medienarbeit, offene Medienprojekte, Peer-to-Peer-Projekte

- Kritik und Diskussion der in den Medienangeboten und Medienhandlungsmustern implizierten Wertorientierungen als Hilfe für den Aufbau einer eigenen Wertrangordnung (Präferenzordnung)

- Kritische Auseinandersetzung mit den gesetzlichen und den pädagogisch gesetzten Begrenzungen, Verboten und Sanktionen des Medienhandelns

- Anregungen zur Reflexion möglicher Folgen des eigenen Medienhandelns für sich, für andere und für die Gesellschaft

- Motivierung zur Konstruktion alternativer Medienhandlungsmuster durch situiertes Lernen mit Medien auf der Basis fallbezogener Aufgaben, Probleme oder Projekte

- Befähigung zur Medienkritik und zur Sensibilisierung für mögliche Gefahren durch problematische Medienangebote oder Medienhandlungsmuster

- Aushandeln und Erweitern der begrenzenden Normen und Regeln mit den Heranwachsenden entsprechend ihren Fähigkeiten im Entwicklungsprozess als Einübung in Verantwortungsfähigkeit und Bereitschaft zur Verantwortungsübernahme im autonomen Medienhandeln.

Viele dieser beispielhaften Massnahmen erscheinen für sich genommen nicht neu, aber sie bekommen im Zusammenhang einer Kontextsteuerung einen anderen Stellenwert, wenn die Komponenten dieser Kontextsteuerung immer wieder neu aufeinander abgestimmt werden. Schwierigkeiten ergeben sich aus der enormen Komplexität der miteinander interagierenden psychischen, sozialen und medialen Sinnsysteme sowie 
aus Konflikten mit anderen gesellschaftlichen Teilsystemen (Wirtschaft, Politik, Arbeitswelt), die ganz andere Sinnorientierungen für das Medienhandeln anbieten. Aber die Möglichkeiten einer kontextuellen Steuerung des Medienhandelns der Heranwachsenden im Spannungsfeld von Rahmensetzung, Autonomiegewährung und pädagogischer Begleitung eröffnen nicht nur neue Lern-, Entwicklungs- und Bildungschancen, sondern auch neue Ansatzpunkte und Wege, um Schwierigkeiten, Probleme und Gefährdungen des Aufwachsens in einer digitalen Lebenswelt zu überwinden. Entscheidend für Medienschutz und Medienpädagogik ist die Anerkennung und Berücksichtigung der Selbstregulation des Medienhandelns der Heranwachsenden und ihrer Doppelfunktion für den einzelnen und die relevanten Umweltsysteme. Damit wird ein Angriff auf die Selbstbestimmung der Person vermieden, die letztlich pädagogisch unverfügbar ist. Eine entwicklungsförderliche Ausrichtung dieser Regulationsprozesse kann jedoch nur auf der Basis tragfähiger sozialer Beziehungen und einer positiven pädagogischen Atmosphäre gelingen. Bei allen Bemühungen um eine mediale Gestaltung der alltagweltlichen Kontexte wird es letztlich darauf ankommen, inwieweit eine Rückbindung der virtuellen Medienwelten der Kinder und Jugendlichen durch persönliche Bindungen an ihr soziales Umfeld und ihre reale Lebenswelt als letzten fundamentalen und Sinn stiftenden Bezugsrahmen für den Entwicklungsprozess gelingt.

\section{Literatur}

Ala-Mutka, Kirsti. 2009. Review of Learning in ICT-Enabled Networks and Communities. Research Centre, Luxemburg: European Commission. https://doi. org/10.2791/29644.

Aufenanger, Stefan. 2008. «Quo vadis Medienpädagogik?»In Medienkompetenz. Zauberwort oder Leerformel des Jugendmedienschutzes, herausgegeben von Thomas Dörken-Kucharz, 61-68. Baden-Baden: Nomos.

Bateson, Gregory. 1990. Geist und Natur. Eine notwendige Einheit. 2. Aufl. Frankfurt/M: Suhrkamp.

Baum, Christoph Georg. 2008. Jugendmedienschutz als Staatsaufgabe. 1. Aufl. Bd. 6o. Materialien zur rechtswissenschaftlichen Medien- und Informationsforschung. Baden-Baden: Nomos Verlagsgesellschaft mbH \& Co. KG. https://doi. org/10.5771/9783845205632. 
Bosch, Dorit. 2007. Die "Regulierte Selbstregulierung” im JugendmedienschutzStaatsvertrag. Eine Bewertung des neuen Aufsichtsmodells anhand verfassungsund europarechtlicher Vorgaben. Frankfurt am Main, Berlin, Bern, Bruxelles, New York, Oxford, Wien: Peter Lang.

Büeler, Xaver. 1994. System Erziehung. Ein bio-psycho-soziales Modell. Bern, Stuttgart, Wien: Haupt.

Bundesländer der Bundesrepublik Deutschland. 2016. Staatsvertrag über den Schutz der Menschenwürde und den Jugendschutz in Rundfunk und Telemedien (Jugendmedienschutz-Staatsvertrag - JMStV). Bd. 2271. http://www.landesrecht-bw.de/jportal/?quelle=jlink\&query=JMedienSchStVtrG+BW\&psml=bsb awueprod.psml\&max $=$ true\&aiz $=$ true.

Castells, Manuel. 2001. Der Aufstieg der Netzwerkgesellschaft. 1. Aufl. Bd. 1. 3 Bd. Das Informationszeitalter. Opladen: Leske+Budrich. https://doi.org/10.1007/978-3322-97534-8.

Doelker, Christian. 2002. "Umwelt als Information - Information als Umwelt. Aufwachsen in der Mediengesellschaft». In Was bieten die Medien? Was braucht die Gesellschaft?, herausgegeben von Thomas Gruber. München: Bayerischer Rundfunk.

Eickelmann, Birgit. 2010. «Digitale Medien in Schule und Unterricht erfolgreich implementieren. Eine empirische Analyse aus Sicht der Schulentwicklungsforschung.» Empirische Erziehungswissenschaft. 19. Münster: Waxmann.

Esposito, Elena. 2010. «Ästhetik und Spiel». In Mensch und Medien: Philosophische und sozialwissenschaftliche Perspektiven, herausgegeben von Manuela Pietraß und Rüdiger Funiok, 159-77. Wiesbaden: VS Verlag für Sozialwissenschaften. https://doi.org/10.1007/978-3-531-92244-7_8.

Feierabend, Sabine, Theresa Plankenhorn, und Thomas Rathgeb. 2017. «FIM-Studie 2016. Familie, Interaktion, Medien Untersuchung zur Kommunikation und Mediennutzung in Familien». Herausgegeben von Medienpädagogischer Forschungsverbund Südwest (mpfs). Medienpädagogischer Forschungsverbund Südwest (LFK, LMK). http://www.mpfs.de/fileadmin/files/Studien/ KIM/2016/KIM_2016_Web-PDF.pdf.

Feierabend, Sabine, Thomas Rathgeb, Hediye Kheredmand, und Stephan Glöckler. 2020. «JIM-Studie 2019. Jugend, Information, Medien. Basisuntersuchung zum Medienumgang 12- bis 19-Jähriger». Herausgegeben von Medienpädagogischer Forschungsverbund Südwest (mpfs). Medienpädagogischer Forschungsverbund Südwest (LFK, LMK). https://www.mpfs.de/fileadmin/files/ Studien/JIM/2019/JIM_2019.pdf.

Feierabend, Sabine, Thomas Rathgeb, und Theresa Reutter. 2019. "KIM-Studie 2018. Kindheit, Internet, Medien. Basisstudie zum Medienumgang 6- bis 13-Jähriger in Deutschland». Herausgegeben von Medienpädagogischer Forschungsverbund Südwest (mpfs). Medienpädagogischer Forschungsverbund Südwest (LFK, LMK). https://www.mpfs.de/fileadmin/files/Studien/KIM/2018/ KIM-Studie_2018_web.pdf. 
Fleischer, Jane. 2018. Erwachsenwerden als Prozess mediatisierter Sozialisation. Wie junge Menschen mit Hilfe online verfügbarer Informationen eigene Entwicklungsaufgaben bearbeiten. Baden-Baden: Nomos.

Hans-Bredow-Institut für Medienforschung, Hrsg. 2014. «Systematisierung der Problemlagen bei der Mediennutzung Minderjähriger: Herausforderungen für den Jugendmedienschutz». In Aufwachsen mit digitalen Medien. Monitoring aktueller Entwicklungen in den Bereichen Medienerziehung und Jugendschutz. Bd. 1. Berichte. Hamburg: Hans-Bredow-Institut für Medienforschung. https:// www.hans-bredow-institut.de/uploads/media/Publikationen/cms/media/724 6c53bcaobbae2a4d3374e8e3be65c64a6be4c.pdf.

Hartmann, Maren, und Andreas Hepp. 2010. Die Mediatisierung der Alltagswelt. Wiesbaden: VS.

Hasebrink, Uwe. 2014. «Die kommunikative Figuration von Familien: Medien, Kommunikation und Informationstechnologie im Familienalltag». In Die Zukunft der Familie: Anforderungen an Familienpolitik und Familienwissenschaft, herausgegeben von Marina Rupp, Olaf Kapella, und Norbert F. Schneider, 22541. Opladen; Berlin; Toronto: Budrich. https://doi.org/10.2307/j.ctvdfolkz.18.

Hasebrink, Uwe, Hermann-Dieter Schröde, und Gerlinde Schumacher. 2012. «Kinder- und Jugendmedienschutz aus der Sicht der Eltern. Ergebnisse einer repräsentativen Elternbefragung». Media Perspektiven 1: 18-30. https://www. ard-werbung.de/media-perspektiven/fachzeitschrift/2012/artikel/kinderund-jugendmedienschutz-aus-der-sicht-der-eltern/.

Hepp, Andreas, und Uwe Hasebrink. 2014. «Kommunikative Figurationen - ein Ansatz zur Analyse der Transformation mediatisierter Gesellschaften und Kulturen». In Von der Gutenberg-Galaxis zur Google-Galaxis. Alte und neue Grenzvermessungen nach 50 Jahren DGPuK, herausgegeben von Birgit Stark, Oliver Quiring, und Nikolaus Jackob. Konstanz: UVK.

Initiative D21. 2016. Sonderstudie "Schule digital". Lehrwelt, Lernwelt, Lebenswelt: Digitale Bildung im Dreieck Schülerinnen - Eltern - Lehrkräfte. https://initiatived21.de/app/uploads/2017/01/d21_schule_digital2016.pdf.

Kammerl, Rudolf, und Michaela Kramer. 2016. «The changing media environment and its impact on socialization processes in families》. Studies in Communication Sciences 16 (1): 21-27. https://doi.org/10.1016/j.scoms.2016.04.004.

$\mathrm{KMK}$ - Kultusministerkonferenz. 2016. «Bildung in der digitalen Welt. Strategie der Kultusministerkonferenz». Herausgegeben von Sekretariat der Kultusministerkonferenz. Kultusministerkonferenz. https://www.kmk.org/fileadmin/ Dateien/pdf/PresseUndAktuelles/2016/Bildung_digitale_Welt_Webversion. pdf.

Koenig, Christoph Jan. 2011. «Bildung im Netz: Analyse und bildungstheoretische Interpretation der neuen kollaborativen Praktiken in offenen Online-Communities». Dissertation, Darmstadt: Technische Universität. http://tuprints.ulb. tu-darmstadt.de/2641/.

Koziol, Klaus. 2000. Die TYrannei der mediengerechten Lösung. Kopäd Verlag. 
Krotz, Friedrich. 2001. Die Mediatisierung kommunikativen Handelns: Der Wandel von Alltag und sozialen Beziehungen, Kultur und Gesellschaft durch die Medien. Wiesbaden: Westdeutscher Verlag. https://doi.org/10.1007/978-3-322-90411-9.

Leschke, Rainer. 2012. «Geschlossene Gesellschaft. Zum kulturellen Regime von Medientechnologien». In Grenzenlose Enthüllungen. Medien zwischen Öffnung und Schließung, herausgegeben von Uta Rußmann, Andreas Beinsteiner, und Heike Ortner, 53-71. Innsbruck: Innsbruck University Press. https://doi. org/10.25969/mediarep/1723.

Luhmann, Niklas. 1991. Soziale Systeme: Grundriss einer allgemeinen Theorie. Bd. 4. Frankfurt/M: Suhrkamp.

Luhmann, Niklas. 1996. Die Realität der Massenmedien. Opladen: Westdeutscher Verlag.

Niesyto, Horst. 2011. Keine Bildung ohne Medien: Positionen, Personen, Programm und Perspektiven. München: kopaed.

Piaget, Jean. 1980. Theorien und Methoden der modernen Erziehung. Frankfurt/M: Fischer.

Pietraß, Manuela. 2014. "Was heißt „Medialitätsbewußtsein”? Eine Ausdeutung des Berichts des BMBF "Kompetenzen in einer digital geprägten Kultur"». merz. medien + erziehung 58 (4): 45-49.

Retzke, Catharina. 2007. Präventiver Jugendmedienschutz: eine Untersuchung des Jugendschutzgesetzes und des Jugendmedienschutz-Staatsvertrags unter besonderer Berücksichtigung des Systems der regulierten Selbstregulierung und der innerstaatlichen und gemeinschaftsrechtlichen Kompetenzabgrenzung. Reihe Wirtschaft und Recht. Sierke Verlag.

Ruckdeschel, Patrick Georg. 2015. «Strukturanalyse des Videospiels. Handlungsorganisation und Semantisierung. Wie Menschen mit Maschinen spielen.» München: kopaed.

Rusch, Gebhard. 1999. «Eine Kommunikationstheorie für kognitive Systeme». In Konstruktivismus in der Medien- und Kommunikationswissenschaft, herausgegeben von Gebhard Rusch und Siegfried J. Schmidt. Frankfurt/M: Suhrkamp.

Rusch, Gebhard. 2007. «Mediendynamik. Explorationen zur Theorie des Medienwandels». Navigationen - Zeitschrift für Medien- und Kulturwissenschaften 7 (1): 13-93. https://doi.org/10.25969/mediarep/2006.

Schill, Wolfgang. 2008. Integrative Medienerziehung in der Grundschule.

Schmidt, Siegfried. 2000. Kalte Faszination. Medien, Kultur, Wissenschaft in der Mediengesellschaft. Weilerswist: Velbrück Wissenschaft.

Schmidt, Siegfried J. 1998. «Medien. Die Kopplung von Kommunikation und Kognition». In Medien, Computer, Realität. Wirklichkeitsvorstellungen und neue Medien, herausgegeben von Sybille Krämer, 55-72. Frankfurt/M: Suhrkamp.

Schmidt, Siegfried J. 2004. «Subjekte - Aktanten zwischen kognitiver Autonomie und sozialer Orientierung». In Subjekttheorien interdisziplinär. Diskussionsbeiträge aus Sozialwissenschaften, Philosophie und Neurowissenschaften, herausgegeben von Grundmann Matthias und Raphael Beer, 135-53. Münster: Lit Verlag. 
Schulz-Zander, Renate. 2005. «Innovativer Unterricht mit Informationstechnologien - Ergebnisse der SITES M2». In Schulentwicklung und Schulwirksamkeit: Systemsteuerung, Bildungschancen und Entwicklung der Schule: 30 Jahre Institut für Schulentwicklungsforschung, herausgegeben von Heinz Günter Holtappels, 264-76. Weinheim: Juventa.

SINUS-Institut Heidelberg. 2015. DIVSI Ug-Studie. Kinder in der digitalen Welt. Herausgegeben von Deutsches Institut für Vertrauen und Sicherheit im Internet (DIVSI). Hamburg: Deutsches Institut für Vertrauen und Sicherheit im Internet. https://www.divsi.de/wp-content/uploads/2015/06/Ug-Studie-DIVSIweb.pdf.

Spanhel, Dieter. 1990. Jugendliche vor dem Bildschirm. 2. Aufl. Weinheim: Deutscher Studienverlag.

Spanhel, Dieter. 1999a. Integrative Medienerziehung in der Hauptschule. Ein Entwicklungsprojekt auf der Grundlage responsiver Evaluation. München: KoPäd.

Spanhel, Dieter. 1999b. «Vom Text zum Kontext: Überlegungen zu einer medienpädagogischen Theorie auf systemtheoretischer Grundlage». In Medien lesen. Der Textbegriff in der Medienwissenschaft, herausgegeben von Daniel Amman, Heinz Moser, und Roger Vaissière, 274-95. Zürich: Verlag Pestalozzianum.

Spanhel, Dieter. 2005. "Medienpädagogik unter anthropologischen Aspekten. Konsequenzen für die Schule». In Medien und Menschen. Medienphilosophische und medienanthropologische Aspekte der Medienbildung, herausgegeben von Jens Winkel, 8:25-42. Lehrerausbildung und Schule in der Diskussion. Paderborn: Paderborner Lehrerausbildungszentrum. http://plaz.uni-paderborn. de/fileadmin/plaz/Plaz_Organisation/Schriftenreihe__PLAZ-Forum_/Winkel2005.pdf.

Spanhel, Dieter. 2006. Medienerziehung. Bd. 3. Handbuch Medienpädagogik. Stuttgart: Klett-Cotta.

Spanhel, Dieter. 2010a. «Bildung in der Mediengesellschaft». In Medienbildung in neuen Kulturräumen: Die deutschprachige und britische Diskussion, herausgegeben von Ben Bachmair, 45-58. Wiesbaden: VS Verlag für Sozialwissenschaften. https://doi.org/10.1007/978-3-531-92133-4_3.

Spanhel, Dieter. 2010b. «Entwicklung und Erziehung unter den Bedingungen von Medialität». In Mensch und Medien: Philosophische und sozialwissenschaftliche Perspektiven, herausgegeben von Manuela Pietraß und Rüdiger Funiok, 65-89. Wiesbaden: VS Verlag für Sozialwissenschaften. https://doi.org/10.1007/978-3531-92244-7_4.

Spanhel, Dieter. 2010c. «Erziehung und Entwicklung unter den Bedingungen von Medialität». In Mensch und Medien. Philosophische und sozialwissenschaftliche Perspektiven, herausgegeben von Manuela Pietraß und Rüdiger Funiok, 65-90. Wiesbaden: VS Verlag für Sozialwissenschaften. https://doi.org/10.1007/978-3531-92244-7_4. 
Spanhel, Dieter. 2013a. «Der Prozess der Identitätsbildung in mediatisierten Alltagswelten». In Medienwelten im Wandel: Kommunikationswissenschaftliche Positionen, Perspektiven und Konsequenzen, herausgegeben von Christine W. Wijnen, Sascha Trültzsch, und Christina Ortner, 79-93. Wiesbaden: Springer Fachmedien. https://doi.org/10.1007/978-3-531-19049-5_7.

Spanhel, Dieter. 2013b. «Sozialisation in mediatisierten Lebenswelten». merz I Wissenschaft 57 (6 Aufwachsen in komplexen Medienwelten): 30-43.

Spanhel, Dieter. 2014. «Der Prozess der Medienbildung auf der Grundlage von Entwicklung, Lernen und Erziehung». In Perspektiven der Medienbildung, herausgegeben von Winfried Marotzki und Norbert Meder, 121-48. Medienbildung und Gesellschaft. Wiesbaden: Springer Fachmedien. https://doi. org/10.1007/978-3-658-03529-7_6.

Spanhel, Dieter. 2015. «Geschichte der Medienpädagogik seit 1980». In Geschichte der Medienpädagogik in Deutschland, herausgegeben von Von Gross, Meister Friederike, M. Dorothee, und Uwe Sander, 94-130. Weinheim; Basel: Beltz Juventa. https://www.beltz.de/fachmedien/erziehungs_und_sozialwissenschaften/buecher/produkt_produktdetails/15109-die_geschichte_der_medienpaedagogik_in_deutschland.html.

Spanhel, Dieter. 2017. «Mediale Bildungsräume - Spielräume der Freiheit in realen und virtuellen Lebenswelten?» MedienPädagogik. Zeitschrift für Theorie und Praxis der Medienbildung, Nr. Einzelbeiträge: 1-18. https://doi.org/10.21240/ mpaed/00/2017.03.02.X.

Spanhel, Dieter. 2018. «Medienkritik aus pädagogischer Perspektive. Kritik der medialen Bedingungen des Aufwachsens unserer Kinder und Jugendlichen». In Medienkritik im digitalen Zeitalter, herausgegeben von Horst Niesyto und Heinz Moser, 11:109-24. Schriftenreihe Medienpädagogik interdisziplinär. München: KoPäd.

Spanhel, Dieter. 2020. «Kinder, Jugendliche und junge Erwachsene in digitalisierten Lernwelten». In Handbuch Soziale Arbeit und Digitalisierung, herausgegeben von Nadia Kutscher, Thomas Ley, Udo Seelmeyer, Friederike Siller, Angela Tillmann, und Isabel Zorn, 101-14. Weinheim, Basel: Beltz, Juventa. https:// content-select.com/de/portal/media/download_oa/9783779952589/?client_ id $=406$.

Stapf, Ingrid. 2018. «Kindliche Selbstbestimmung in der digital vernetzten Welt. Kinderrechte zwischen Schutz, Befähigung und Partizipation mit Blick auf „evolving capacities"». merz - medien + erziehung 62 (6 Kinder, Medien): 7-18.

Theunert, Helga. 2005. «Medien als Orte informellen Lernens im Prozess des Heranwachsens». In Kompetenzerwerb von Kindern und Jugendlichen im Schulalter, herausgegeben von Cathleen Grunert, Werner Helsper, Merle Hummrich, Helga Theunert, und Ingrid Gogolin, 3:175-300. Materialien zum Zwölften Kinder-und Jugendbericht. München: Verlag Deutsches Jugendinstitut. https://www.dji.de/fileadmin/user_upload/bibs/Vergriffene_Buecher_Open_Access/Sachverstaendigenkommission\%2012Bericht\%20Kompetenzerwerb\%20 von\%20Kindern\%20und\%20Jugendlichen\%20im\%20Schulalter.pdf. 
Thye, Iris. 2013. Kommunikation und Gesellschaft - systemtheoretisch beobachtet. Sprache, Schrift, einseitige Massen- und digitale Online-Medien. Wiesbaden: Springer VS. https://doi.org/10.1007/978-3-658-00439-2.

Tulodziecki, Gerhard, und Bardo Herzig. 2002. Computer und Internet im Unterricht. Medienpädagogische Grundlagen und Beispiele im Unterricht. Berlin: Cornelsen.

Tulodziecki, Gerhard, Bardo Herzig, und Silke Grafe. 2010. Medienbildung in Schule und Unterricht. Bad Heilbrunn: Klinkhardt.

Wagner, Ulrike, und Christa Gebel. 2015. «Medienerziehung in der Familie unter den Bedingungen von Mediatisierung». In Jahrbuch Medienpädagogik 12: Kinder und Kindheit in der digitalen Kultur, herausgegeben von Kai-Uwe Hugger, Angela Tillmann, Stefan Iske, Johannes Fromme, Petra Grell, und Theo Hug, 12:11-28. Jahrbuch Medienpädagogik. Wiesbaden: Springer Fachmedien Wiesbaden. https://doi.org/10.1007/978-3-658-09809-4_2.

Wagner, Ulrike, Christa Gebel, Claudia Lampert, und Landesanstalt für Medien Nordrhein-Westfalen, Hrsg. 2013. Zwischen Anspruch und Alltagsbewältigung: Medienerziehung in der Familie. Schriftenreihe Medienforschung der Landesanstalt für Medien Nordrhein-Westfalen 72. Berlin: Vistas-Verl. [u.a.].

Weidenmann, Bernd. 2006. «Lernen mit Medien». In Pädagogische Psychologie: ein Lehrbuch, herausgegeben von Andreas Krapp und B. Weidenmann, 5., vollst. überarb. Aufl., 423-76. Weinheim: Beltz PVU.

Willke, Helmut. 1991. Systemtheorie. 3. überarb. Aufl. Stuttgart, New York: Fischer.

Willke, Helmut. 2005. Symbolische Systeme. Grundriss einer soziologischen Theorie. Weilerswist: Velbrück-Wissenschaft. 